\title{
Toric Geometry of the Regular Convex Polyhedra
}

\author{
Fiammetta Battaglia and Elisa Prato \\ Dipartimento di Matematica e Informatica “U. Dini”, Università di Firenze, Viale Morgagni 67/A, 50134 Firenze, Italy \\ Correspondence should be addressed to Elisa Prato; elisa.prato@unifi.it
}

Received 30 July 2016; Accepted 31 January 2017; Published 23 March 2017

Academic Editor: Frédéric Mynard

Copyright (C) 2017 Fiammetta Battaglia and Elisa Prato. This is an open access article distributed under the Creative Commons Attribution License, which permits unrestricted use, distribution, and reproduction in any medium, provided the original work is properly cited.

We describe symplectic and complex toric spaces associated with the five regular convex polyhedra. The regular tetrahedron and the cube are rational and simple, the regular octahedron is not simple, the regular dodecahedron is not rational, and the regular icosahedron is neither simple nor rational. We remark that the last two cases cannot be treated via standard toric geometry.

\section{Introduction}

Among the five regular convex polyhedra, the regular tetrahedron and the cube are examples of simple rational convex polytopes. To these, the standard smooth toric geometry applies, in both the symplectic and complex category. From the symplectic viewpoint, in fact, the regular tetrahedron and the cube satisfy the hypotheses of the Delzant theorem [1], and it is easily seen that they correspond, respectively, to $S^{7} / S^{1}$ and $S^{2} \times S^{2} \times S^{2}$. From the complex viewpoint, on the other hand, the toric variety associated with the regular tetrahedron is $\mathbb{C P}^{3}$ while $\mathbb{C P}^{1} \times \mathbb{C P}^{1} \times \mathbb{C P}^{1}$ corresponds to the cube (see, e.g., [2, Section 1.5]); these can also be obtained as quotients by a complex version of the Delzant procedure described by Audin in [3, Chapter VI].

The regular octahedron is still rational, but it is no longer simple. The toric variety associated with the regular octahedron is however well known and is described, for example, by Fulton in [2, Section 1.5]; it can also be obtained as a complex quotient by applying the Cox construction [4, Theorem 2.1].

The regular dodecahedron, on the other hand, is simple but it is the first of the five regular convex polyhedra that is not rational. It is shown by Prato in [5] that, by applying her extension of the Delzant procedure to the case of general simple convex polytopes [6], one can associate with the regular dodecahedron a symplectic toric quasifold. Quasifolds are a generalization of manifolds and orbifolds: they are not necessarily Hausdorff and they are locally modeled by the quotient of a manifold modulo the action of a discrete group.

In this article, we recall all of the above and we complete the picture, first of all, by associating with the regular dodecahedron a complex toric quasifold. We do so by applying a generalization, given by the authors in [7], of the procedure described by Audin in [3, Chapter VI] to the case of general simple convex polytopes. As in the smooth case, the symplectic and complex quotients can be identified [7, Theorem 3.2], endowing the corresponding toric quasifold with a Kähler structure.

We go on to address the case of the regular icosahedron. From the toric viewpoint, this is certainly the most complicated of the five regular convex polyhedra, since it is neither simple nor rational. However, we can apply generalizations by Battaglia and Prato [8] and Battaglia $[9,10]$ of toric quasifolds $[6,7]$ and of the Cox construction [4] to arbitrary convex polytopes; this allows us to associate with the regular icosahedron, in both the symplectic and complex category, a space that is stratified by quasifolds. As for all 3-dimensional polytopes, here there are only zero-dimensional singular strata and an open dense regular stratum. Moreover, by $[10$, Theorem 3.3], the symplectic and complex quotients can be identified, endowing the regular stratum with the structure of a Kähler quasifold.

Notice, finally, that we are still missing a symplectic toric space corresponding to the regular octahedron; this too can be found by applying Battaglia's work on arbitrary convex 
polytopes. What we get here is a space that is stratified by symplectic manifolds (see [9, Remark 6.6]); moreover, by [10, Theorem 3.3], this symplectic quotient can be identified with the complex quotient, and the regular stratum is Kähler.

The article is structured as follows: in Section 2 we recall a few necessary facts on convex polytopes; in Section 3 we recall from $[6,7]$ how to construct symplectic and complex toric quasifolds from simple convex polytopes; in Section 4 we recall from $[9,10]$ the construction of the symplectic and complex toric spaces corresponding to arbitrary convex polytopes; finally, in Sections 5, 6, 7, and 8 we describe the symplectic and complex toric spaces corresponding to the five regular convex polyhedra.

\section{Facts on Convex Polytopes}

Consider a dimension $n$ convex polytope $\Delta \subset\left(\mathbb{R}^{n}\right)^{*}$.

Definition 1 (simple polytope). $\Delta$ is said to be simple if each of its vertices is contained in exactly $n$ facets.

Assume now that $\Delta$ has $d$ facets. Then there exist elements $X_{1}, \ldots, X_{d}$ in $\mathbb{R}^{n}$ and real numbers $\lambda_{1}, \ldots, \lambda_{d}$ such that

$$
\Delta=\bigcap_{j=1}^{d}\left\{\mu \in\left(\mathbb{R}^{n}\right)^{*} \mid\left\langle\mu, X_{j}\right\rangle \geq \lambda_{j}\right\} .
$$

Let us consider the open faces of $\Delta$. They can be described as follows. For each such face $F$ there exists a possibly empty subset $I_{F} \subset\{1, \ldots, d\}$ such that

$$
F=\left\{\mu \in \Delta \mid\left\langle\mu, X_{j}\right\rangle=\lambda_{j} \text { iff } j \in I_{F}\right\} .
$$

A partial order on the set of all open faces of $\Delta$ is defined by setting $F \leq F^{\prime}$ (we say $F$ contained in $F^{\prime}$ ) if $F \subseteq \overline{F^{\prime}}$. Notice that $F \leq F^{\prime}$ if and only if $I_{F^{\prime}} \subset I_{F}$. The polytope $\Delta$ is the disjoint union of its open faces. Let $r_{F}=\operatorname{card}\left(I_{F}\right)$; we have the following definitions.

Definition 2. A $p$-dimensional open face $F$ of the polytope is said to be singular if $r_{F}>n-p$.

Definition 3. A $p$-dimensional open face $F$ of the polytope is said to be regular if $r_{F}=n-p$.

Remark 4. Let $F$ be a $p$-dimensional singular face in $\left(\mathbb{R}^{n}\right)^{*}$, then $p<n-2$. Therefore any polytope in $\left(\mathbb{R}^{2}\right)^{*}$ is simple and the singular faces of a nonsimple polytope in $\left(\mathbb{R}^{3}\right)^{*}$ are vertices.

We refer the reader to Ziegler's book [11] for additional basic facts on convex polytopes. We now go on to recall what is meant by quasilattice and quasirational polytope.

Definition 5 (quasilattice). A quasilattice in $\mathbb{R}^{n}$ is the $\mathbb{Z}$-span of a set of $\mathbb{R}$-spanning vectors, $Y_{1}, \ldots, Y_{q}$, of $\mathbb{R}^{n}$.

Notice that $\operatorname{Span}_{\mathbb{Z}}\left\{Y_{1}, \ldots, Y_{q}\right\}$ is a lattice if and only if it is generated by a basis of $\mathbb{R}^{n}$.
Definition 6 (quasirational polytope). Let $Q$ be a quasilattice in $\mathbb{R}^{n}$. A convex polytope $\Delta \subset\left(\mathbb{R}^{n}\right)^{*}$ is said to be quasirational with respect to the quasilattice $Q$ if the vectors $X_{1}, \ldots, X_{d}$ in (1) can be chosen in $Q$.

Remark that each polytope in $\left(\mathbb{R}^{n}\right)^{*}$ is quasirational with respect to the quasilattice $Q$ that is generated by the elements $X_{1}, \ldots, X_{d}$ in (1). We note that if $X_{1}, \ldots, X_{d}$ can be chosen inside a lattice, then the polytope is rational.

\section{The Simple Case}

Let $\Delta \subset\left(\mathbb{R}^{n}\right)^{*}$ be an $n$-dimensional simple convex polytope. We are now ready to recall from [6] and [7] the construction of the symplectic and complex toric quasifolds associated with $\Delta$. For the definition and main properties of symplectic and complex quasifolds we refer the reader to [6,12] and [7]. For the purposes of this article, we will restrict our attention to the special case $n=3$. We begin by remarking that both constructions rely on the notion of quasitorus, which we recall.

Definition 7 (quasitorus). Let $Q$ be a quasilattice in $\mathbb{R}^{3}$. We call quasitorus of dimension 3 the group and quasifold $D^{3}=$ $\mathbb{R}^{3} / Q$.

Notice that, if the quasilattice is a lattice, we obtain the classical notion of torus. The quasilattice $Q$ also acts naturally on $\mathbb{C}^{3}$ :

$$
\begin{aligned}
Q \times \mathbb{C}^{3} & \longrightarrow \mathbb{C}^{3} \\
(A, X+i Y) & \longmapsto(X+A)+i Y .
\end{aligned}
$$

Therefore, in the complex category we have the following.

Definition 8 (complex quasitorus). Let $Q$ be a quasilattice in $\mathbb{R}^{3}$. We call complex quasitorus of dimension 3 the group and complex quasifold $D_{\mathbb{C}}^{3}=\mathbb{C}^{3} / Q$.

In analogy with the smooth case, we will say that $D_{\mathbb{C}}^{3}$ is the complexification of $D^{3}$. Assume now that our polytope $\Delta$ is quasirational with respect to a quasilattice $Q$ and write

$$
\Delta=\bigcap_{j=1}^{d}\left\{\mu \in\left(\mathbb{R}^{3}\right)^{*} \mid\left\langle\mu, X_{j}\right\rangle \geq \lambda_{j}\right\}
$$

for some elements $X_{1}, \ldots, X_{d} \in Q$ and some real numbers $\lambda_{1}, \ldots, \lambda_{d}$; again, $d$ here is the number of facets of $\Delta$. Let $\left\{e_{1}, \ldots, e_{d}\right\}$ denote the standard basis of $\mathbb{R}^{d}$ and $\mathbb{C}^{d}$. Consider the surjective linear mapping

$$
\begin{aligned}
\pi: \mathbb{R}^{d} & \longrightarrow \mathbb{R}^{3} \\
e_{j} & \longmapsto X_{j},
\end{aligned}
$$

and its complexification

$$
\begin{aligned}
\pi_{\mathbb{C}}: & \mathbb{C}^{d} \longrightarrow \mathbb{C}^{3} \\
e_{j} & \longmapsto X_{j} .
\end{aligned}
$$


Consider the quasitori $D^{3}=\mathbb{R}^{3} / Q$ and $D_{\mathbb{C}}^{3}=\mathbb{C}^{3} / Q$. The mappings $\pi$ and $\pi_{\mathbb{C}}$ each induce group epimorphisms

$$
\begin{gathered}
\Pi: T^{d}=\frac{\mathbb{R}^{d}}{\mathbb{Z}^{d}} \longrightarrow D^{3}, \\
\Pi_{\mathbb{C}}: T_{\mathbb{C}}^{d}=\frac{\mathbb{C}^{d}}{\mathbb{Z}^{d}} \longrightarrow D_{\mathbb{C}}^{3} .
\end{gathered}
$$

We define $N$ to be the kernel of the mapping $\Pi$ and $N_{\mathbb{C}}$ to be the kernel of the mapping $\Pi_{\mathbb{C}}$. Notice that neither $N$ nor $N_{\mathbb{C}}$ are honest tori unless $Q$ is a honest lattice. The Lie algebras of $N$ and $N_{\mathbb{C}}$ are, respectively, $\mathfrak{n}=\operatorname{ker} \pi$ and $\mathfrak{n}_{\mathbb{C}}=\operatorname{ker} \pi_{\mathbb{C}}$. The mappings $\Pi$ and $\Pi_{\mathbb{C}}$ induce isomorphisms

$$
\begin{gathered}
\frac{T^{d}}{N} \longrightarrow D^{3}, \\
\frac{T_{\mathbb{C}}^{d}}{N_{\mathbb{C}}} \longrightarrow D_{\mathbb{C}}^{3} .
\end{gathered}
$$

Let us begin with the symplectic construction. Consider the space $\mathbb{C}^{d}$, endowed with the symplectic form

$$
\omega_{0}=\frac{1}{2 \pi i} \sum_{j=1}^{d} d z_{j} \wedge d \bar{z}_{j}
$$

and the action of the torus $T^{d}=\mathbb{R}^{d} / \mathbb{Z}^{d}$ :

$$
\begin{aligned}
T^{d} \times \mathbb{C}^{d} & \longrightarrow \mathbb{C}^{d} \\
\left(\left(e^{2 \pi i \theta_{1}}, \ldots, e^{2 \pi i \theta_{d}}\right), \underline{z}\right) & \longmapsto\left(e^{2 \pi i \theta_{1}} z_{1}, \ldots, e^{2 \pi i \theta_{d}} z_{d}\right) .
\end{aligned}
$$

This action is effective and Hamiltonian, with moment mapping given by

$$
\begin{aligned}
J: \mathbb{C}^{d} & \longrightarrow\left(\mathbb{R}^{d}\right)^{*} \\
\underline{z} & \longmapsto \sum_{j=1}^{d}\left|z_{j}\right|^{2} e_{j}^{*}+\lambda, \quad \lambda \in\left(\mathbb{R}^{d}\right)^{*} \text { constant. }
\end{aligned}
$$

Choose now $\lambda=\sum_{j=1}^{d} \lambda_{j} e_{j}^{*}$, with $\lambda_{1}, \ldots, \lambda_{d}$ as in (4). Denote by $i$ the Lie algebra inclusion $\mathfrak{n} \rightarrow \mathbb{R}^{d}$ and notice that $\Psi=i^{*} \circ J$ is a moment mapping for the induced action of $N$ on $\mathbb{C}^{d}$. Consider now the orbit space $M_{\Delta}=\Psi^{-1}(0) / N$. Then we have, from [6, Theorem 3.3], the following.

Theorem 9 (generalized Delzant construction). Let $Q$ be a quasilattice in $\mathbb{R}^{3}$ and let $\Delta \subset\left(\mathbb{R}^{3}\right)^{*}$ be a 3-dimensional simple convex polytope that is quasirational with respect to $Q$. Assume that $d$ is the number of facets of $\Delta$ and consider vectors $X_{1}, \ldots, X_{d}$ in $Q$ that satisfy (4). For each $\left(\Delta, Q,\left\{X_{1}, \ldots, X_{d}\right\}\right)$, the orbit space $M_{\Delta}$ is a compact, connected 6-dimensional symplectic quasifold endowed with an effective Hamiltonian action of the quasitorus $D^{3}=\mathbb{R}^{3} / Q$ such that, if $\Phi: M \rightarrow\left(\mathbb{R}^{3}\right)^{*}$ is the corresponding moment mapping, then $\Phi\left(M_{\Delta}\right)=\Delta$.

We say that the quasifold $M_{\triangle}$ with the effective Hamiltonian action of $D^{3}$ is the symplectic toric quasifold associated with $\left(\Delta, Q,\left\{X_{1}, \ldots, X_{d}\right\}\right)$.
Let us now pass to the complex construction. Following the notation of the previous section, consider, for any open face $F$ of $\Delta$, the $T_{\mathbb{C}}^{d}$-orbit

$$
\mathbb{C}_{F}^{d}=\left\{\underline{z} \in \mathbb{C}^{d} \mid z_{j}=0 \text { iff } j \in I_{F}\right\} .
$$

Consider the open subset of $\mathbb{C}^{d}$ given by

$$
\mathbb{C}_{\Delta}^{d}=\bigcup_{F \in \Delta}\left\{\underline{z} \in \mathbb{C}^{d} \mid z_{j} \neq 0 \text { if } j \notin I_{F}\right\} .
$$

Notice that

$$
\mathbb{C}_{\Delta}^{d}=\bigcup_{\mu \in \Delta}\left\{\underline{z} \in \mathbb{C}^{d} \mid z_{j} \neq 0 \text { if } j \notin I_{\mu}\right\},
$$

where $\mu$ ranges over all the vertices of the polytope $\Delta$. Moreover, since the polytope is simple, we have that

$$
\mathbb{C}_{\Delta}^{d}=\bigcup_{F} \mathbb{C}_{F}^{d}
$$

In fact, in this case, $\left\{\underline{z} \in \mathbb{C}^{d} \mid z_{j} \neq 0\right.$ if $\left.j \notin I_{F}\right\}=\cup_{F^{\prime} \geq F} \mathbb{C}_{F^{\prime}}^{d}$. The group $N_{\mathbb{C}}$ acts on the space $\mathbb{C}_{\Delta}^{d}$. Consider the space of orbits $X_{\Delta}=\mathbb{C}_{\Delta}^{d} / N_{\mathbb{C}}$. We then have, from [7, Theorem 2.2], the following.

Theorem 10. Let $Q$ be a quasilattice in $\mathbb{R}^{3}$ and let $\Delta \subset\left(\mathbb{R}^{3}\right)^{*}$ be a 3-dimensional simple convex poly tope that is quasirational with respect to $Q$. Assume that $d$ is the number of facets of $\Delta$ and consider vectors $X_{1}, \ldots, X_{d}$ in $Q$ that satisfy (4). For each $\left(\Delta, Q,\left\{X_{1}, \ldots, X_{d}\right\}\right)$, the corresponding quotient $X_{\Delta}$ is a complex quasifold of dimension 3 , endowed with a holomorphic action of the complex quasitorus $D_{\mathbb{C}}^{3}=\mathbb{C}^{3} / Q$ having a dense open orbit.

We say that the quasifold $X_{\Delta}$ with the holomorphic action of $D_{\mathbb{C}}^{3}$ is the complex toric quasifold associated with $\left(\Delta, Q,\left\{X_{1}, \ldots, X_{d}\right\}\right)$.

Finally, we conclude this section by recalling that the natural embedding

$$
\Psi^{-1}(0) \hookrightarrow \mathbb{C}_{\Delta}^{d}
$$

induces a mapping

$$
\chi: M_{\Delta} \longrightarrow X_{\Delta}
$$

that sends each $N$-orbit to the corresponding $N_{\mathbb{C}}$-orbit. This mapping is equivariant with respect to the actions of the quasitori $D^{3}$ and $D_{\mathbb{C}}^{3}$. Then, under the same assumptions of Theorems 9 and 10, we have, from [7, Theorem 3.2], the following.

Theorem 11. The mapping $\chi: M_{\Delta} \rightarrow X_{\Delta}$ is an equivariant diffeomorphism of quasifolds. Moreover, the induced symplectic form on the complex quasifold $X_{\Delta}$ is Kähler.

For the smooth case see Audin [3, Proposition 3.1.1] but also Guillemin [13, Appendix 1, Theorem 1.4]. 


\section{The Nonsimple Case}

Consider now a nonsimple convex polytope $\Delta \subset\left(\mathbb{R}^{3}\right)^{*}$ and assume that $\Delta$ is quasirational with respect to a quasilattice $Q$. The idea here is to repeat the constructions of the previous section. If we do so, we again find the groups $N$ and $N_{\mathbb{C}}$ and the quasitori $D^{3}$ and $D_{\mathbb{C}}^{3}$, isomorphic to $T^{d} / N$ and $T_{\mathbb{C}}^{d} / N_{\mathbb{C}}$, respectively. However, the symplectic construction produces spaces that are stratified by symplectic quasifolds, while the complex construction yields spaces that are stratified by complex quasifolds. For the exact definitions of these notions we refer the reader to [9, Section 2] and [10, Definition 1.5]. We remark that in the smooth case these definitions yield the classical definition of Goresky and MacPherson [14]. Many of the important features of these stratified structures will be clarified when addressing the relevant examples (see Sections 6 and 8).

Let us consider the symplectic case first. The main difference with respect to the case of simple polytopes is that here there are points in the level set $\Psi^{-1}(0)$ that have isotropy groups of positive dimension; therefore $\Psi^{-1}(0)$ is no longer a smooth manifold. From Proposition 3.3 and Theorems 5.3, $5.10,5.11,6.4$ in [9] we have the following.

Theorem 12 (generalized Delzant construction: nonsimple case). Let $Q$ be a quasilattice in $\mathbb{R}^{3}$ and let $\Delta \subset\left(\mathbb{R}^{3}\right)^{*}$ be a 3-dimensional convex polytope that is quasirational with respect to $Q$. Assume that $d$ is the number of facets of $\Delta$ and consider vectors $X_{1}, \ldots, X_{d}$ in $Q$ that satisfy (4). For each $\left(\Delta, Q,\left\{X_{1}, \ldots, X_{d}\right\}\right)$, the quotient $M_{\Delta}$ is a compact, connected 6-dimensional space stratified by symplectic quasifolds, endowed with an effective continuous action of the quasitorus $D^{3}=\mathbb{R}^{3} / Q$. Moreover, there exists a continuous mapping $\Phi: M \rightarrow\left(\mathbb{R}^{3}\right)^{*}$ such that $\Phi\left(M_{\Delta}\right)=\Delta$. Finally, the restriction of the $D^{3}$-action to each stratum is smooth and Hamiltonian, with moment mapping given by the restriction of $\Phi$.

When the polytope is rational, these quotients are examples of the symplectic stratified spaces described by Sjamaar and Lerman in [15]; in particular, the strata are either manifolds or orbifolds [9, Remark 6.6]. We remark that the nonsimple rational case was addressed also by Burns et al. in $[16,17]$; they gave an in-depth treatment and a classification theorem in the case of isolated singularities.

Let us now examine the complex case. Here, one still considers the open subset $\mathbb{C}_{\Delta}^{d}$ as defined in (13) but while in the simple case the orbits of $\exp (i \mathfrak{n})$ on $\mathbb{C}_{\Delta}^{d}$ were closed, here there are nonclosed orbits. We recall first that $N_{\mathbb{C}}=N A$, where $A=\exp (i \mathfrak{n})$; this actually happens also in the simple case. Then, from [10, Theorem 2.1], we have the following.

Theorem 13 (closed orbits). Let $\underline{z} \in \mathbb{C}_{\Delta}^{d}$. Then $A \cdot \underline{z}$, the A-orbit through $\underline{z}$, is closed if and only if there exists a face $F$ such that $\underline{z}$ is in $\mathbb{C}_{F}^{d}$. Moreover, if $A \cdot \underline{z}$ is nonclosed, then its closure contains one, and only one, closed A-orbit.

Therefore, in order to define a notion of quotient, one defines the following equivalence relation: two points $\underline{z}$ and $\underline{w}$ in $\mathbb{C}_{\Delta}^{d}$ are equivalent with respect to the action of the group $N_{\mathbb{C}}$ if and only if

$$
(N(\overline{A \cdot \underline{z}})) \cap(\overline{A \cdot \underline{w}}) \neq \emptyset,
$$

where the closure is meant in $\mathbb{C}_{\Delta}^{d}$. The space $X_{\Delta}$ is then defined to be the quotient $\mathbb{C}_{\Delta}^{d} / / N_{\mathbb{C}}$ with respect to this equivalence relation. Notice that if the polytope is simple, $\mathbb{C}_{\Delta}^{d}=\cup_{F \in \Delta} \mathbb{C}_{F}^{d}$; thus, by Theorem $13, A$-orbits through points in $\mathbb{C}_{\Delta}^{d}$ are always closed and the quotient $X_{\Delta}$ is just the orbit space endowed with the quotient topology. From [10, Proposition 3.1, Theorem 3.2] we have the following.

Theorem 14. Let $Q$ be a quasilattice in $\mathbb{R}^{3}$ and let $\Delta \subset\left(\mathbb{R}^{3}\right)^{*}$ be a 3-dimensional convex polytope that is quasirational with respect to $Q$. Assume that $d$ is the number of facets of $\Delta$ and consider vectors $X_{1}, \ldots, X_{d}$ in $Q$ that satisfy (4). For each $\left(\Delta, Q,\left\{X_{1}, \ldots, X_{d}\right\}\right)$, the corresponding quotient $X_{\Delta}$ is endowed with a stratification by complex quasifolds of dimension 3. The complex quasitorus $D_{\mathbb{C}}^{3}$ acts continuously on $X_{\Delta}$, with a dense open orbit. Moreover, the restriction of the $D_{\mathbb{C}}^{3}$-action to each stratum is holomorphic.

We remark that when $Q$ is a lattice and the vectors $X_{1}, \ldots, X_{d}$ are primitive in $Q$, the quotient $X_{\Delta}$ coincides with the Cox presentation [4] of the classical toric variety that corresponds to the fan normal to the polytope $\Delta$. As for classical toric varieties, there is a one-to-one correspondence between $p$-dimensional orbits of the quasitorus $D_{\mathbb{C}}^{3}$ and $p$-dimensional faces of the polytope. In particular, the dense open orbit corresponds to the interior of the polytope and the singular strata correspond to singular faces.

We remark that, like in the simple case, the natural embedding $\Psi^{-1}(0) \hookrightarrow \mathbb{C}_{\Delta}^{d}$ induces an identification between symplectic and complex quotients. From [10, Theorem 3.3] we have the following.

Theorem 15. The mapping $\chi_{\Delta}: M_{\Delta} \rightarrow X_{\Delta}$ is a homeomorphism which is equivariant with respect to the actions of $D^{3}$ and $D_{\mathbb{C}}^{3}$, respectively. Moreover, the restriction of $\chi_{\Delta}$ to each stratum is a diffeomorphism of quasifolds. Finally, the induced symplectic form on each stratum is compatible with its complex structure, so that each stratum is Kähler.

We conclude by pointing out that $M_{\Delta} \simeq X_{\Delta}$ has two different kinds of singularities, namely, the stratification and the quasifold structure of the strata. The nonsimplicity of the polytope yields the decomposition in strata of the corresponding topological space, while its nonrationality produces the quasifold structure of the strata and also intervenes in the way the strata are glued to each other. This last feature can be observed only in spaces with strata of positive dimension; this led to a definition of stratification that naturally extends the usual one [9, Section 2]. 


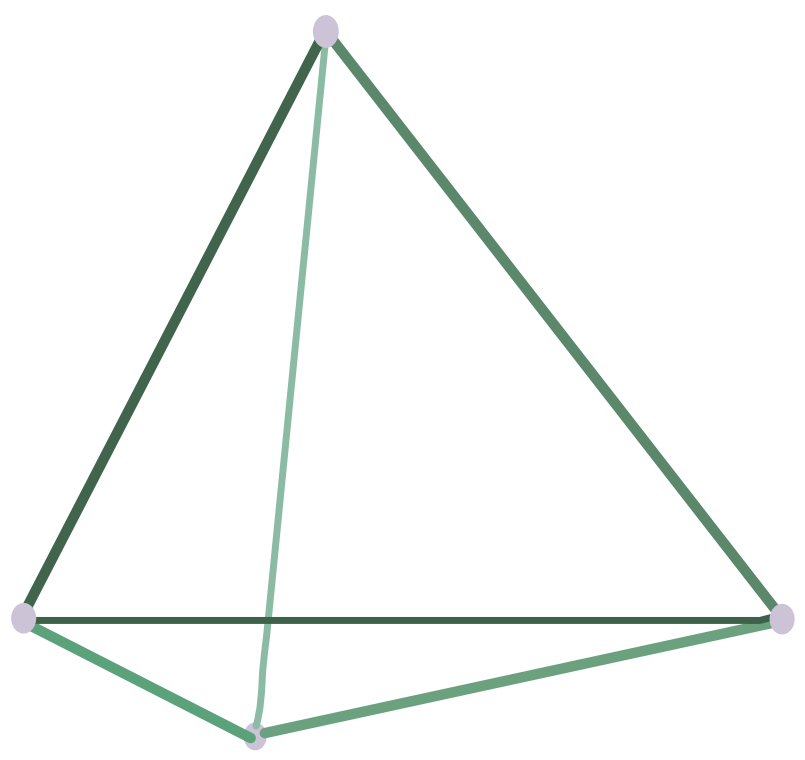

Figure 1: The regular tetrahedron.

\section{Simple and Rational: The Regular Tetrahedron and the Cube}

The regular tetrahedron and the cube are both simple and rational. Let us recall the construction of the corresponding symplectic and complex toric manifolds. We follow the notation of Section 3, which also applies to the smooth case.

Let us begin with the regular tetrahedron $\Delta$ (see Figure 1) having vertices

$$
\begin{aligned}
& v_{1}=(1,1,1), \\
& v_{2}=(1,-1,-1), \\
& v_{3}=(-1,1,-1), \\
& v_{4}=(-1,-1,1) .
\end{aligned}
$$

Consider the sublattice $L$ of $\mathbb{Z}^{3}$ that is generated by the corresponding four vectors

$$
\begin{aligned}
& Y_{1}=(1,1,1), \\
& Y_{2}=(1,-1,-1), \\
& Y_{3}=(-1,1,-1), \\
& Y_{4}=(-1,-1,1) .
\end{aligned}
$$

Notice that $Y_{1}+Y_{2}+Y_{3}+Y_{4}=0$; therefore any three of these four vectors form a basis of $L$. Moreover,

$$
\Delta=\bigcap_{j=1}^{4}\left\{\mu \in\left(\mathbb{R}^{3}\right)^{*} \mid\left\langle\mu, X_{j}\right\rangle \geq-1\right\}
$$

where $X_{j}=Y_{j}, j=1, \ldots, 4$. Thus the regular tetrahedron $\Delta$ satisfies the hypotheses of Delzant's theorem [1] with respect to $L$. From the symplectic viewpoint, it is readily verified here

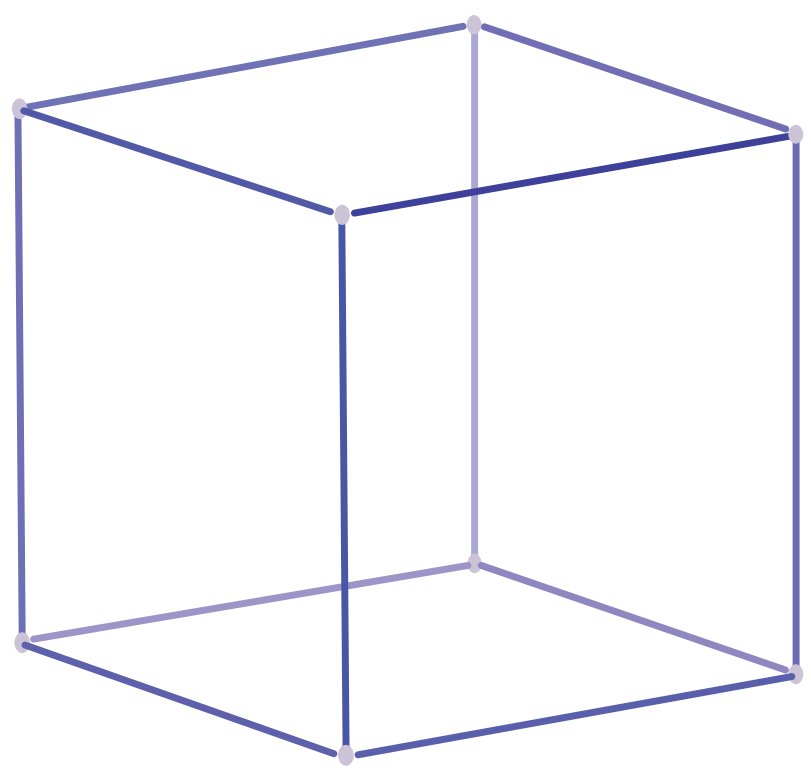

Figure 2: The cube.

that $N=\left\{\left(e^{2 \pi i \theta}, e^{2 \pi i \theta}, e^{2 \pi i \theta}, e^{2 \pi i \theta}\right) \mid \theta \in \mathbb{R}\right\}=S^{1}$ and therefore that $M_{\Delta}$ is given by

$$
\frac{\left\{\left.\underline{z} \in \mathbb{C}^{4}|| z_{1}\right|^{2}+\left|z_{2}\right|^{2}+\left|z_{3}\right|^{2}+\left|z_{4}\right|^{2}=4\right\}}{S^{1}}=\frac{S^{7}}{S^{1}}
$$

where $S^{7}$ denotes the 7 -sphere of radius 2 . From the complex viewpoint, it is easy to see that $\mathbb{C}_{\Delta}^{4}=\mathbb{C}^{4} \backslash\{0\}$, that $N_{\mathbb{C}}=\mathbb{C}^{*}$, and therefore that

$$
X_{\Delta}=\frac{\mathbb{C}^{4} \backslash\{0\}}{\mathbb{C}^{*}}=\mathbb{C} \mathbb{P}^{3}
$$

Consider now the cube $\Delta$ having vertices $( \pm 1, \pm 1, \pm 1$ ) (see Figure 2). Notice that

$$
\Delta=\bigcap_{j=1}^{6}\left\{\mu \in\left(\mathbb{R}^{3}\right)^{*} \mid\left\langle\mu, X_{j}\right\rangle \geq-1\right\}
$$

where $X_{1}=e_{1}, X_{2}=-e_{1}, X_{3}=e_{2}, X_{4}=-e_{2}, X_{5}=e_{3}$, and $X_{6}=-e_{3}$. We can again apply the Delzant procedure, this time relatively to the lattice $\mathbb{Z}^{3}$, and we get that the 3 dimensional group $N$ is given by

$$
\begin{aligned}
& \left\{\left(e^{2 \pi i \theta_{1}}, e^{2 \pi i \theta_{1}}, e^{2 \pi i \theta_{2}}, e^{2 \pi i \theta_{2}}, e^{2 \pi i \theta_{3}}, e^{2 \pi i \theta_{3}}\right) \mid \theta_{1}, \theta_{2}, \theta_{3}\right. \\
& \quad \in \mathbb{R}\}=S^{1} \times S^{1} \times S^{1} \subset T^{6}
\end{aligned}
$$

and, therefore, the symplectic toric manifold $M_{\Delta}$ is given by

$$
\begin{aligned}
& \frac{\left\{\left.\underline{z} \in \mathbb{C}^{6}|| z_{1}\right|^{2}+\left|z_{2}\right|^{2}=2,\left|z_{3}\right|^{2}+\left|z_{4}\right|^{2}=2,\left|z_{5}\right|^{2}+\left|z_{6}\right|^{2}=2\right\}}{N} \\
& =S^{2} \times S^{2} \times S^{2}
\end{aligned}
$$




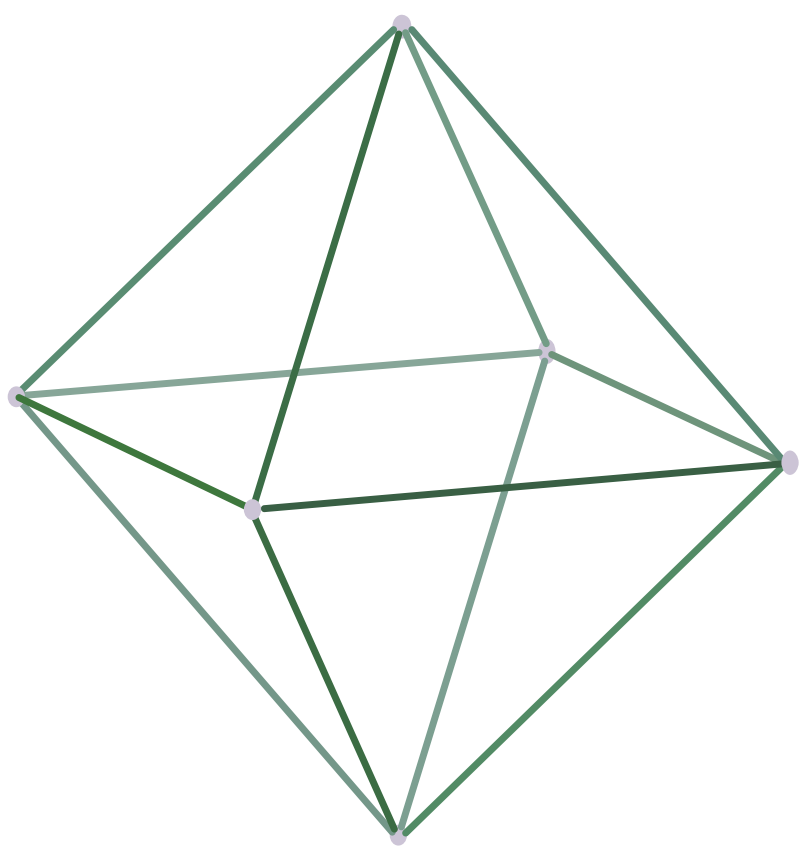

FIgURE 3: The regular octahedron.

where the $S^{2}$ 's have all radius $\sqrt{2}$. The corresponding complex toric manifold $X_{\Delta}$, on the other hand, is given by

$$
\begin{gathered}
\frac{\left(\left(\mathbb{C}^{2} \backslash\{0\}\right) \times\left(\mathbb{C}^{2} \backslash\{0\}\right) \times\left(\mathbb{C}^{2} \backslash\{0\}\right)\right)}{\left(\mathbb{C}^{*} \times \mathbb{C}^{*} \times \mathbb{C}^{*}\right)} \\
=\mathbb{C P}^{1} \times \mathbb{C P}^{1} \times \mathbb{C} \mathbb{P}^{1} .
\end{gathered}
$$

This provides an elementary example of a general fact: the symplectic toric manifold depends on the polytope, while the complex toric manifold only depends on the fan that is normal to the polytope. For instance, let us consider the cube having vertices $( \pm a, \pm a, \pm a)$, with $a$ a positive real number, with the same vectors $X_{1}, \ldots, X_{6}$ as above. Then the corresponding symplectic toric manifold is the product of three spheres of radius $\sqrt{2 a}$. In conclusion, the symplectic structure varies, while the complex toric manifold remains the same.

\section{Nonsimple and Rational: \\ The Regular Octahedron}

Let us consider the regular octahedron $\Delta$ that is dual to the cube of Section 5 (see Figure 3). Its vertices are given by $v_{1}=e_{1}, v_{2}=-e_{1}, v_{3}=e_{2}, v_{4}=-e_{2}, v_{5}=e_{3}, v_{6}=-e_{3}$. This polytope is rational with respect to the lattice $L$ defined in Section 5.

In fact

$$
\Delta=\bigcap_{j=1}^{8}\left\{\mu \in\left(\mathbb{R}^{3}\right)^{*} \mid\left\langle\mu, X_{j}\right\rangle \geq-1\right\}
$$

TABLE 1

\begin{tabular}{lc}
\hline Plane & Vertices \\
\hline$H_{1}$ & $\left(v_{2}, v_{4}, v_{6}\right)$ \\
$H_{2}$ & $\left(v_{2}, v_{3}, v_{5}\right)$ \\
$H_{3}$ & $\left(v_{1}, v_{4}, v_{5}\right)$ \\
$H_{4}$ & $\left(v_{1}, v_{3}, v_{6}\right)$ \\
$H_{5}$ & $\left(v_{1}, v_{3}, v_{5}\right)$ \\
$H_{6}$ & $\left(v_{1}, v_{4}, v_{6}\right)$ \\
$H_{7}$ & $\left(v_{2}, v_{3}, v_{6}\right)$ \\
$H_{8}$ & $\left(v_{2}, v_{4}, v_{5}\right)$ \\
\hline
\end{tabular}

where $X_{i}=Y_{i}, X_{4+i}=-Y_{i}, i=1, \ldots, 4$. However $\Delta$ is not simple. More precisely, consider the planes

$$
H_{i}=\left\{\mu \in\left(\mathbb{R}^{3}\right)^{*} \mid\left\langle\mu, X_{j}\right\rangle=-1\right\},
$$

$i=1, \ldots, 8$, containing the eight facets of the octahedron. Notice that each such plane contains exactly three vertices, as shown in Table 1.

Furthermore, each vertex is given by the intersection of four planes:

$$
\begin{aligned}
& v_{1}=H_{3} \cap H_{4} \cap H_{5} \cap H_{6}, \\
& v_{2}=H_{1} \cap H_{2} \cap H_{7} \cap H_{8}, \\
& v_{3}=H_{2} \cap H_{4} \cap H_{5} \cap H_{7}, \\
& v_{4}=H_{1} \cap H_{3} \cap H_{6} \cap H_{8}, \\
& v_{5}=H_{2} \cap H_{3} \cap H_{5} \cap H_{8}, \\
& v_{6}=H_{1} \cap H_{4} \cap H_{6} \cap H_{7} .
\end{aligned}
$$

Therefore, each vertex is a singular face of $\Delta$. If we apply the generalized Delzant construction we find that $N=\exp (\mathfrak{n})$, with $\mathfrak{n}$ given by

$$
\begin{aligned}
& \left\{\left(\theta_{1}, \theta_{2}, \theta_{3}, \theta_{4}, \theta_{1}+s, \theta_{2}+s, \theta_{3}+s, \theta_{4}+s\right) \mid \theta_{1}, \theta_{2}, \theta_{3}, \theta_{4},\right. \\
& \quad s \in \mathbb{R}\} .
\end{aligned}
$$

The level set $\Psi^{-1}(0)$ for the moment mapping $\Psi$ with respect to the induced $N$-action on $\mathbb{C}^{8}$ is therefore given by the compact subset of points $\underline{z}$ in $\mathbb{C}^{8}$ such that

$$
\begin{aligned}
& \left|z_{1}\right|^{2}+\left|z_{5}\right|^{2}=2, \\
& \left|z_{2}\right|^{2}+\left|z_{6}\right|^{2}=2, \\
& \left|z_{3}\right|^{2}+\left|z_{7}\right|^{2}=2, \\
& \left|z_{4}\right|^{2}+\left|z_{8}\right|^{2}=2, \\
& \left|z_{3}\right|^{2}+\left|z_{4}\right|^{2}=\left|z_{5}\right|^{2}+\left|z_{6}\right|^{2} .
\end{aligned}
$$

Notice that the $N$-action has isotropies of dimension 1 at the $T^{8}$-orbits corresponding to the 6 (singular) vertices. For 
example, consider the first vertex: $v_{1}=H_{3} \cap H_{4} \cap H_{5} \cap H_{6}$. The corresponding orbit in $\Psi^{-1}(0)$ is

$$
\left\{\left.\underline{z} \in \mathbb{C}^{8}|| z_{1}\right|^{2}=2,\left|z_{2}\right|^{2}=2,\left|z_{7}\right|^{2}=2,\left|z_{8}\right|^{2}=2\right\}
$$

with isotropy $\left\{\left(1,1, e^{2 \pi i t}, e^{2 \pi i t}, 1,1, e^{2 \pi i t}, e^{2 \pi i t}\right) \mid t \in \mathbb{R}\right\}$. Away from the orbits corresponding to the vertices, the level set is smooth. By Theorem 12, the orbit space

$$
M_{\Delta}=\frac{\Psi^{-1}(0)}{N}
$$

is an elementary example of symplectic toric space stratified by manifolds. The six orbits in $\Psi^{-1}(0)$ corresponding to the vertices yield six singular points in the quotient $M_{\Delta}$. Their complement is the regular stratum of $M_{\Delta}$, which is a smooth symplectic manifold.

From the complex viewpoint, by (14), the set $\mathbb{C}_{\Delta}^{8}$ is the union of 6 open sets, each of which corresponds to a vertex. The Cox quotient $X_{\Delta}=\mathbb{C}_{\Delta}^{8} / / N_{\mathbb{C}}$ is an elementary example of complex toric space stratified by manifolds. The quotients $M_{\Delta}$ and $X_{\Delta}$ can be identified according to Theorem 15 .

Let us briefly describe $M_{\Delta}$ as a symplectic toric stratified space. By Theorem 12, the action of the torus $D^{3}=\mathbb{R}^{3} / L$ on $M_{\Delta}$ is effective and continuous. Moreover, the image of the continuous mapping $\Phi=\left(\pi^{*}\right)^{-1} \circ J$ is exactly $\Delta$ and each singular point is sent by $\Phi$ to the corresponding vertex. The action of the torus $D^{3}=\mathbb{R}^{3} / L$ on the regular stratum is smooth and Hamiltonian, and the mapping $\Phi$ is the moment mapping with respect to the $D^{3}$-action. By Theorem 15, the regular stratum is a 6-dimensional Kähler manifold [9, Remark 6.6].

We now describe the local structure of the regular stratum and of the stratification around the singular points. Consider, for example, the vertex $v_{1}$. We recall that the singular point $m_{1} \in M_{\Delta}$ corresponding to $v_{1}$ is given by

$$
\frac{\left\{\left.\underline{z} \in \mathbb{C}^{8}|| z_{1}\right|^{2}=2,\left|z_{2}\right|^{2}=2,\left|z_{7}\right|^{2}=2,\left|z_{8}\right|^{2}=2\right\}}{N} .
$$

We compute a symplectic chart for the regular stratum in a neighborhood of $m_{1}$. Consider the open subset $U$ given by

$$
\begin{aligned}
& \left\{\left.\left(u_{3}, u_{4}, u_{5}\right) \in \mathbb{C}^{3} \backslash\{0\}|| u_{5}\right|^{2}<\left|u_{3}\right|^{2}+\left|u_{4}\right|^{2}<2\right. \\
& \left.\quad+\left|u_{5}\right|^{2},\left|u_{i}\right|^{2}<2, i=3,4,5\right\}
\end{aligned}
$$

and the mapping

$$
\begin{aligned}
& \tau: U \longrightarrow\left\{\underline{z} \in \Psi^{-1}(0) \mid z_{i} \neq 0, i=1,2,6,7,8,\left(z_{3}, z_{4}, z_{5}\right) \neq(0,0,0)\right\} \\
&\left(u_{3}, u_{4}, u_{5}\right) \longmapsto \\
&\left(\tau_{1}(\underline{u}), \tau_{2}(\underline{u}), u_{3}, u_{4}, u_{5}, \tau_{6}(\underline{u}), \tau_{7}(\underline{u}), \tau_{8}(\underline{u})\right),
\end{aligned}
$$

where

$$
\begin{aligned}
& \tau_{1}(\underline{u})=\sqrt{2-\left|u_{5}\right|^{2}}, \\
& \tau_{2}(\underline{u})=\sqrt{2-\left|u_{3}\right|^{2}-\left|u_{4}\right|^{2}+\left|u_{5}\right|^{2}}, \\
& \tau_{6}(\underline{u})=\sqrt{\left|u_{3}\right|^{2}+\left|u_{4}\right|^{2}-\left|u_{5}\right|^{2}}, \\
& \tau_{7}(\underline{u})=\sqrt{2-\left|u_{3}\right|^{2}}, \\
& \tau_{8}(\underline{u})=\sqrt{2-\left|u_{4}\right|^{2}} .
\end{aligned}
$$

The mapping $\tau$ is a homeomorphism (see Proof of [9, Theorem 5.3]). The pair $\left(\left(U, \omega_{0}\right), \tau\right)$, where $\omega_{0}$ is the standard symplectic structure of $\mathbb{C}^{3}$, defines a symplectic chart for the regular part in a neighborhood of $m_{1}$. To find other charts, repeat the procedure by replacing the indices $\{3,4,5\}$ with any triple of indices $\{i, j, k\}$ contained in one of the six sets

$$
\begin{aligned}
& \{3,4,5,6\},\{1,2,7,8\},\{2,4,5,7\},\{1,3,6,8\},\{2,3,5,8\}, \\
& \{1,4,6,7\} .
\end{aligned}
$$

We recall that, according to (30), each of these sets corresponds to a different vertex. We need at least 12 charts to obtain a symplectic atlas for the regular stratum.

The regular part can also be seen as a complex manifold. Consider the open subset

$$
V=\mathbb{C}^{3} \backslash\{0\}
$$

and the mapping

$$
\begin{array}{ccc}
\tau_{\mathbb{C}}: \quad & \longrightarrow\left\{\underline{z} \in \mathbb{C}_{\Delta}^{8} \mid z_{i} \neq 0, i=1,2,6,7,8,\left(z_{3}, z_{4}, z_{5}\right) \neq(0,0,0)\right\} \\
\left(u_{3}, u_{4}, u_{5}\right) & \longmapsto & \left(1,1, u_{3}, u_{4}, u_{5}, 1,1,1\right) .
\end{array}
$$

The mapping $\tau_{\mathbb{C}}$ is a homeomorphism. The pair $\left(V, \tau_{\mathbb{C}}\right)$ defines a complex chart for the regular part in a neighborhood of the singular point $x_{1}=\chi\left(m_{1}\right)$. Again, a complex atlas can be obtained by considering all the triples of indices $\{i, j, k\}$ contained in one of the six sets (39). Similarly to [7, Example 3.8], one can compute the local expression of the 
mapping $\chi$ as a diffeomorphism from $U$ to $V$ and find the local expression for the Kähler form on $V$.

Let us now give an example of the existence of nonclosed $A$-orbits for nonsimple polytopes. Consider a point in $\mathbb{C}_{\Delta}^{8}$ that does not lie in any $\mathbb{C}_{F}^{8}$; for instance,

$$
\begin{aligned}
\underline{z}=\left(z_{1}: z_{2}: z_{3}: 0: 0: 0: z_{7}: z_{8}\right), & \\
& z_{1}, z_{2}, z_{3}, z_{7}, z_{8} \neq 0 .
\end{aligned}
$$

Take the element $a(t)=\left(1,1, e^{-2 \pi t}, e^{-2 \pi t}, e^{2 \pi t}, e^{2 \pi t}, 1,1\right) \in$ $A$. When $t$ tends to $+\infty, a(t) \cdot \underline{z}$ tends to $\left(z_{1}: z_{2}: 0: 0: 0: 0: z_{7}: z_{8}\right)$, which therefore lies in the closure of the orbit $A \cdot \underline{z}$. Notice that, by Theorem 13 , the orbit through $\left(z_{1}: z_{2}: 0: 0: 0: 0: z_{7}: z_{8}\right)$ is the only closed orbit contained in $\overline{A \cdot z}$.

Let us now show that the quotient $M_{\Delta} \simeq X_{\Delta}$ is a stratified space in the sense of [14] with isolated singularities. More precisely we show that, in this case, a neighborhood of each singular point $m_{j}$, with $j=1, \ldots, 6$, can be identified with a cone over a manifold, called the link of $m$. We consider the first vertex; the same argument applies to the others. Consider the cone stemming from $v_{1}$

$$
\mathscr{C}=\bigcap_{j=3}^{6}\left\{\mu \in\left(\mathbb{R}^{3}\right)^{*} \mid\left\langle\mu, X_{j}\right\rangle \geq-1\right\} .
$$

The complex and symplectic toric spaces corresponding to this cone are $X_{\mathscr{C}}=\mathbb{C}^{4} / / N_{\mathbb{C}}(\mathscr{C})$ and

$$
M_{\mathscr{C}}=\frac{\left\{\left.\underline{z} \in \mathbb{C}^{4}|| z_{3}\right|^{2}+\left|z_{4}\right|^{2}=\left|z_{5}\right|^{2}+\left|z_{6}\right|^{2}\right\}}{N(\mathscr{C})},
$$

where

$$
N(\mathscr{C})=\exp \{(s, s,-s,-s) \mid s \in \mathbb{R}\} .
$$

We now see explicitly that $M_{\mathscr{C}}$ and $X_{\mathscr{C}}$ are cones (diffeomorphic by the proof of [10, Theorem 3.3]) which are local models for our toric space $M_{\Delta} \simeq X_{\Delta}$ near the singular point $m_{1}=\chi^{-1}\left(x_{1}\right)$. This will prove that $M_{\Delta} \simeq X_{\Delta}$ is a stratified space. Following the recipes given in [10, Sections 3.2, 3.4], the link for $m_{1}=\chi^{-1}\left(x_{1}\right)$ can be found by cutting the cone $\mathscr{C}$ with the $y z$-plane; this gives the square $\Delta_{L}$ of vertices $(0, \pm 1, \pm 1)$. The 5 -dimensional real link $L$ is then given by

$$
\begin{aligned}
& \frac{\left\{\left.\underline{z} \in \mathbb{C}^{4}|| z_{3}\right|^{2}+\left|z_{4}\right|^{2}=\left|z_{5}\right|^{2}+\left|z_{6}\right|^{2}=2\right\}}{N(\mathscr{C})} \\
& \simeq \frac{\left(S^{3} \times S^{3}\right)}{S^{1}}
\end{aligned}
$$

where the spheres $S^{3}$ have both radius $\sqrt{2}$ and $S^{1}$ acts with weights +1 on the first two coordinates and with weights -1 on the last two. From the complex point of view, we have

$$
L_{\mathbb{C}}=\frac{\left(\left(\mathbb{C}^{2} \backslash\{0\}\right) \times\left(\mathbb{C}^{2} \backslash\{0\}\right)\right)}{\left(N_{\mathbb{C}}(\mathscr{C}) \exp \{i(s, s, s, s) \mid s \in \mathbb{R}\}\right)} .
$$

Notice that the inclusion

$$
\left\{\left.\underline{z} \in \mathbb{C}^{4}|| z_{3}\right|^{2}+\left|z_{4}\right|^{2}=\left|z_{5}\right|^{2}+\left|z_{6}\right|^{2}=2\right\} \hookrightarrow \mathbb{C}^{4}
$$

induces a diffeomorphism $L \simeq L_{\mathbb{C}}$. By applying the Delzant procedure to the square $\Delta_{L}$, with respect to the normal vectors $\pm e_{2}, \pm e_{3}$, one finds $N\left(\Delta_{L}\right)=\exp \{(\mathrm{s}, s, t, t) \mid s, t \in \mathbb{R}\}$. Thus the symplectic toric manifold $M_{\Delta_{L}}$ is given by

$$
\frac{\left\{\left.\underline{z} \in \mathbb{C}^{4}|| z_{3}\right|^{2}+\left|z_{4}\right|^{2}=\left|z_{5}\right|^{2}+\left|z_{6}\right|^{2}=2\right\}}{N\left(\Delta_{L}\right)} \simeq S^{2} \times S^{2},
$$

where the spheres $S^{2}$ also have radius $\sqrt{2}$. We follow the terminology of [15] and refer to this manifold as the symplectic link. Correspondingly, the complex link in the sense of [14, p. 15] is given by

$$
X_{\Delta_{L}}=\frac{\left(\left(\mathbb{C}^{2} \backslash\{0\}\right) \times\left(\mathbb{C}^{2} \backslash\{0\}\right)\right)}{N_{\mathbb{C}}\left(\Delta_{L}\right)} \simeq \mathbb{C P}^{1} \times \mathbb{C P}^{1} .
$$

Notice that

$$
\mathbb{C}^{4} / / N_{\mathbb{C}}(\mathscr{C}) \backslash[0]=\frac{\left(\left(\mathbb{C}^{2} \backslash\{0\}\right) \times\left(\mathbb{C}^{2} \backslash\{0\}\right)\right)}{N_{\mathbb{C}}(\mathscr{C})} ;
$$

this shows that the cone $X_{\mathscr{C}}$ is a complex cone over the complex link. Finally, remark that our space $M_{\mathscr{C}} \simeq X_{\mathscr{C}}$, without the singular point that corresponds to the cone apex, projects first onto the real link $L \simeq L_{\mathbb{C}}$ and then onto the symplectic and complex links as follows:

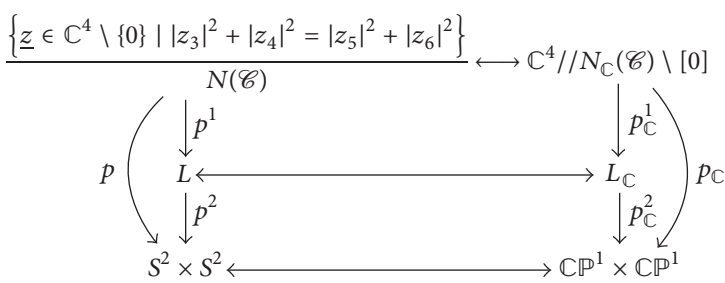

On the left hand side, $p^{1}([z])=\left[r z_{1}: r z_{2}: r z_{3}: r z_{4}\right]$, with $r=\left((1 / 2)\left(\left|z_{3}\right|^{2}+\left|z_{4}\right|^{2}\right)\right)^{-1 / 2}, p^{2}$ is the natural projection, and $p$ is the composite. On the right hand side the mappings are the natural projections.

The fiber of the projections $p^{1}$ and $p_{\mathbb{C}}^{1}$ is $\mathbb{R}_{>0}$, the fiber of the projections $p^{2}$ and $p_{\mathbb{C}}^{2}$ is

$$
\frac{N\left(\Delta_{L}\right)}{N(\mathscr{C})} \simeq \exp \{(t, t, t, t) \mid t \in \mathbb{R}\} \simeq S^{1},
$$

and the fiber of the projections $p$ and $p_{\mathbb{C}}$ is

$$
\begin{aligned}
\frac{N_{\mathbb{C}}\left(\Delta_{L}\right)}{N_{\mathbb{C}}(\mathscr{C})} & \simeq \exp \{(u, u, u, u) \mid u \in \mathbb{C}\} \simeq \mathbb{R}_{>0} \times S^{1} \\
& \simeq \mathbb{C}^{*} .
\end{aligned}
$$

Therefore, $X_{\mathscr{C}} \simeq M_{\mathscr{C}}$ is a real cone over $L_{\mathbb{C}} \simeq L$ and a complex cone over the symplectic/complex link. Finally, notice that $L / S^{1}$ gives the symplectic link.

We now show that $M_{\mathscr{C}}$ and $X_{\mathscr{C}}$ are local models for $M_{\Delta}$ and $X_{\Delta}$, respectively. Consider, in the cone

$$
\left\{\left.\underline{z} \in \mathbb{C}^{4} \backslash\{0\}|| z_{3}\right|^{2}+\left|z_{4}\right|^{2}=\left|z_{5}\right|^{2}+\left|z_{6}\right|^{2}\right\} / N(\mathscr{C}),
$$


the neighborhood $\mathscr{C}_{1}$ of the apex given by the set of points such that $\left|z_{i}\right|^{2}<2, i=3,4,5,6$. The continuous mapping

$$
\begin{gathered}
\mathscr{C}_{1} \\
{\left[z_{3}: z_{4}: z_{5}: z_{6}\right] \longmapsto\left[\sqrt{2-\left|z_{3}\right|^{2}}: \sqrt{2-\left|z_{4}\right|^{2}}: z_{3}: z_{4}: z_{5}: z_{6}: \sqrt{2-\left|z_{7}\right|^{2}}: \sqrt{2-\left|z_{8}\right|^{2}}\right]}
\end{gathered}
$$

is a symplectomorphism on the regular part and sends singular point to singular point. On the other hand, the continuous mapping

$$
\begin{aligned}
& \mathbb{C}^{4} / / N_{\mathbb{C}}^{\mathscr{C}} \longrightarrow\left\{\underline{z} \in \mathbb{C}^{8} \mid z_{i} \neq 0, i=1,2,7,8\right\} / / N_{\mathbb{C}} \subset \mathbb{C}_{\Delta}^{8} / / N_{\mathbb{C}} \\
& {\left[z_{3}: z_{4}: z_{5}: z_{6}\right] \longmapsto \quad\left[1: 1: z_{3}: z_{4}: z_{5}: z_{6}: 1: 1\right]}
\end{aligned}
$$

is a biholomorphism on the regular part and also sends singular point to singular point.

\section{Simple and Nonrational: The Regular Dodecahedron}

Let $\phi=(1+\sqrt{5}) / 2$ be the golden ratio and remark that it satisfies the equation $\phi=1+1 / \phi$. Let $\Delta$ be the regular dodecahedron having vertices

$$
\begin{aligned}
& ( \pm 1, \pm 1, \pm 1), \\
& \left(0, \pm \phi, \pm \frac{1}{\phi}\right), \\
& \left( \pm \frac{1}{\phi}, 0, \pm \phi\right), \\
& \left( \pm \phi, \pm \frac{1}{\phi}, 0\right)
\end{aligned}
$$

(see Figure 4). The polytope $\Delta$ is simple but not rational. However, it is quasirational with respect to the quasilattice $P$, known in physics as the simple icosahedral lattice [18], that is generated by the following vectors in $\mathbb{R}^{3}$ :

$$
\begin{aligned}
& Y_{1}=\left(\frac{1}{\phi^{2}}, \frac{1}{\phi}, 0\right), \\
& Y_{2}=\left(0, \frac{1}{\phi^{2}}, \frac{1}{\phi}\right), \\
& Y_{3}=\left(\frac{1}{\phi}, 0, \frac{1}{\phi^{2}}\right), \\
& Y_{4}=\left(-\frac{1}{\phi^{2}}, \frac{1}{\phi}, 0\right),
\end{aligned}
$$

$$
\begin{aligned}
& Y_{5}=\left(0,-\frac{1}{\phi^{2}}, \frac{1}{\phi}\right), \\
& Y_{6}=\left(\frac{1}{\phi}, 0,-\frac{1}{\phi^{2}}\right) .
\end{aligned}
$$

In fact, a straightforward computation shows that

$$
\Delta=\bigcap_{j=1}^{12}\left\{\mu \in\left(\mathbb{R}^{3}\right)^{*} \mid\left\langle\mu, X_{j}\right\rangle \geq-1\right\}
$$

where $X_{i}=Y_{i}, X_{6+i}=-Y_{i}, i=1, \ldots, 6$. Notice that the vectors $X_{1}, \ldots, X_{12}$ point to the twelve vertices of the regular icosahedron that is dual to $\Delta$ (see Figures 5 and 6 ).

We recall from [5] that if we apply the generalized Delzant construction to $\Delta$ with respect to the quasilattice $P$ and the vectors $X_{1}, \ldots, X_{12}$, we get $N=\exp (\mathfrak{n}), \mathfrak{n}$ being the 9 dimensional subspace of $\mathbb{R}^{12}$ that is spanned by the vectors

$$
\begin{gathered}
e_{1}+e_{7}, \\
e_{2}+e_{8}, \\
e_{3}+e_{9}, \\
e_{4}+e_{10}, \\
e_{5}+e_{11}, \\
e_{6}+e_{12}, \\
e_{1}+e_{2}-\phi\left(e_{3}+e_{4}\right), \\
e_{2}+e_{3}-\phi\left(e_{1}+e_{5}\right), \\
e_{1}+e_{3}-\phi\left(e_{2}+e_{6}\right) .
\end{gathered}
$$




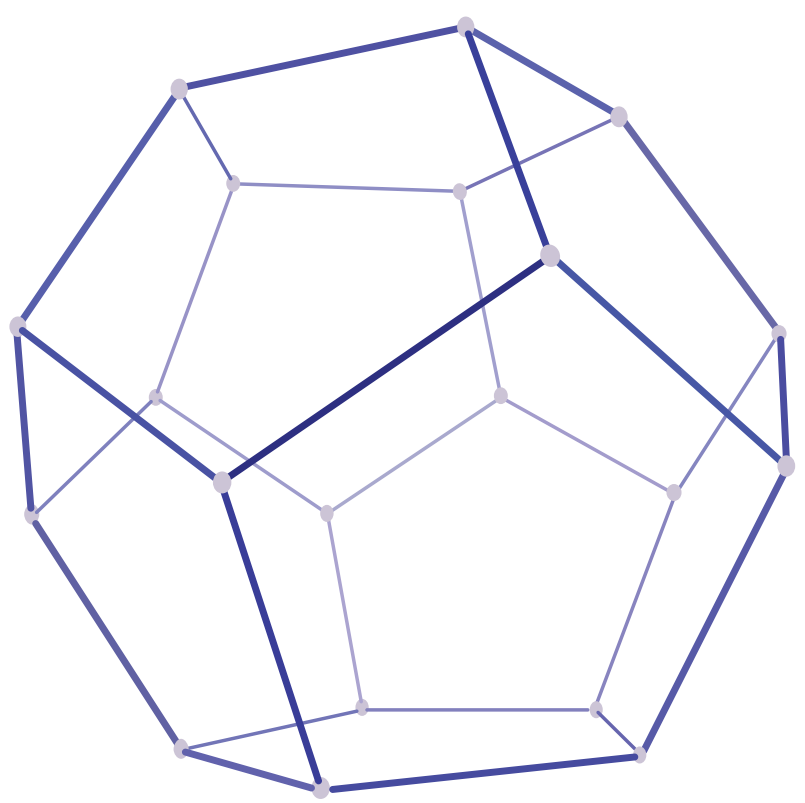

FIgURE 4: The regular dodecahedron.

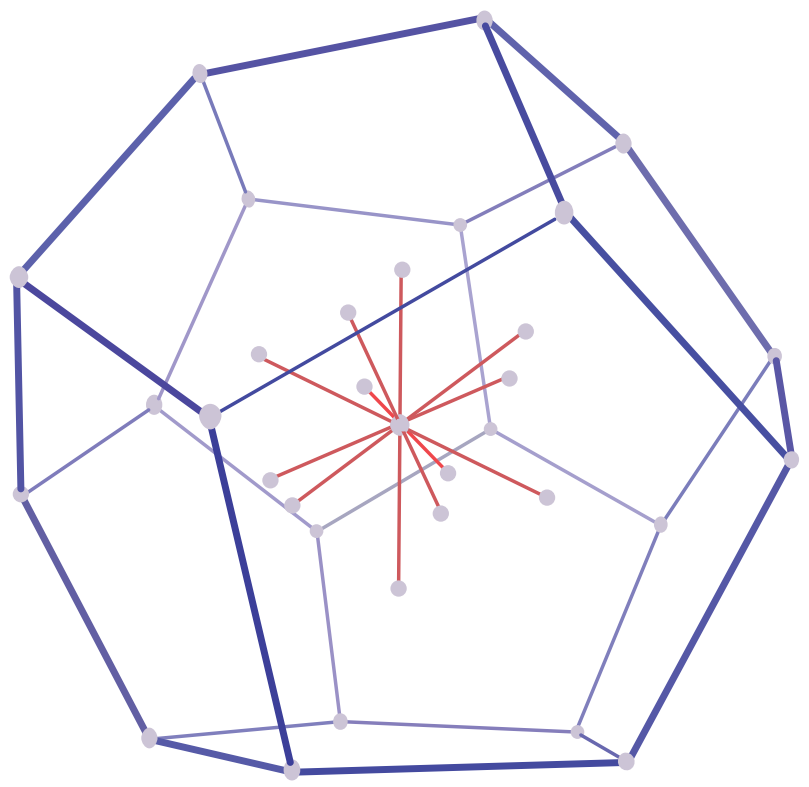

Figure 5: The vectors $X_{1}, \ldots, X_{12}$.

The level set $\Psi^{-1}(0)$ for the moment mapping $\Psi$ with respect to the induced $N$-action on $\mathbb{C}^{12}$ is therefore given by the compact subset of points $z$ in $\mathbb{C}^{12}$ such that

$$
\begin{aligned}
& \left|z_{1}\right|^{2}+\left|z_{7}\right|^{2}=2, \\
& \left|z_{2}\right|^{2}+\left|z_{8}\right|^{2}=2, \\
& \left|z_{3}\right|^{2}+\left|z_{9}\right|^{2}=2,
\end{aligned}
$$

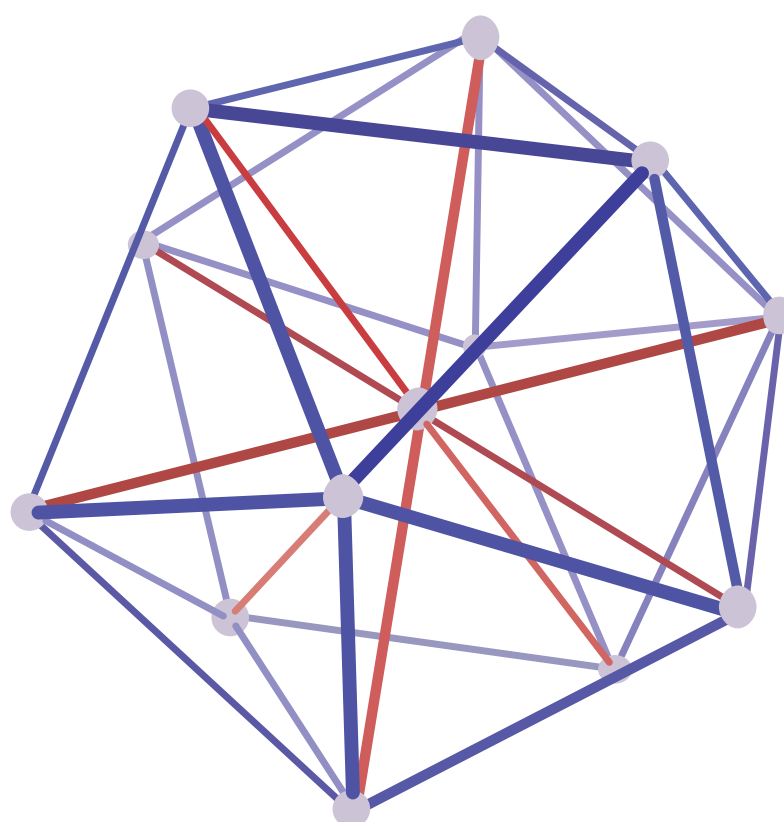

Figure 6: The dual icosahedron.

$$
\begin{aligned}
\left|z_{4}\right|^{2}+\left|z_{10}\right|^{2} & =2, \\
\left|z_{5}\right|^{2}+\left|z_{11}\right|^{2} & =2, \\
\left|z_{6}\right|^{2}+\left|z_{12}\right|^{2} & =2, \\
\left|z_{1}\right|^{2}+\left|z_{2}\right|^{2}-\phi\left(\left|z_{3}\right|^{2}+\left|z_{4}\right|^{2}\right) & =-\frac{2}{\phi}, \\
\left|z_{2}\right|^{2}+\left|z_{3}\right|^{2}-\phi\left(\left|z_{1}\right|^{2}+\left|z_{5}\right|^{2}\right) & =-\frac{2}{\phi}, \\
\left|z_{1}\right|^{2}+\left|z_{3}\right|^{2}-\phi\left(\left|z_{2}\right|^{2}+\left|z_{6}\right|^{2}\right) & =-\frac{2}{\phi} .
\end{aligned}
$$

The quotient $M_{\Delta}=\Psi^{-1}(0) / N$ is a symplectic quasifold; it has an atlas made of 20 charts, each corresponding to a different fixed point of the $D^{3}$-action; we refer the reader to [5] for a description of one of them.

From the complex viewpoint, the complex toric quasifold corresponding to the dodecahedron, with the choice of the same vectors $X_{j}(j=1, \ldots, 12)$ above, is given by the quotient

$$
X_{\Delta}=\frac{\mathbb{C}_{\Delta}^{12}}{N_{\mathbb{C}}}
$$

where $\mathbb{C}_{\Delta}^{12}$ is the open subset of $\mathbb{C}^{12}$ given by the union of the 20 open subsets defined in (14). The symplectic and complex quotients can be identified by Theorem 11 .

Let us describe a chart for the complex toric quasifold $X_{\Delta}$ around the fixed point corresponding to the vertex 
$(-1,-1,-1)$. Let $\widetilde{V}=\mathbb{C}^{3}$ and consider the following slice of $\mathbb{C}_{\Delta}^{12}$ that is transversal to the $N_{\mathbb{C}^{-}}$-orbits

$$
\begin{aligned}
& \widetilde{\tau}_{\mathbb{C}}: \quad \widetilde{V} \quad \longrightarrow\left\{\underline{w} \in \mathbb{C}_{\Delta}^{12} \mid w_{i} \neq 0, i=4, \ldots, 12\right\} \\
& \left(z_{1}, z_{2}, z_{3}\right) \longmapsto \quad\left(z_{1}, z_{2}, z_{3}, 1, \ldots, 1\right) .
\end{aligned}
$$

The mapping $\widetilde{\tau}_{\mathbb{C}}$ induces a homeomorphism

$$
\begin{array}{ccc}
\tau_{\mathbb{C}}: & \frac{\widetilde{V}}{\Gamma} \quad \longrightarrow \quad & U \\
{\left[\left(z_{1}, z_{2}, z_{3}\right)\right]} & \longmapsto\left[\widetilde{\tau}_{\mathbb{C}}\left(z_{1}, z_{2}, z_{3}\right)\right],
\end{array}
$$

where the open subset $V$ of $X_{\Delta}$ is the quotient

$$
\frac{\left\{\underline{w} \in \mathbb{C}_{\Delta}^{12} \mid w_{i} \neq 0, i=4, \ldots, 12\right\}}{N_{\mathbb{C}}}
$$

and the discrete group $\Gamma$ is given by

$$
\left\{\left(e^{2 \pi i \phi(h+l)}, e^{2 \pi i \phi(h+k)}, e^{2 \pi i \phi(k+l)}\right) \in T^{3} \mid h, k, l \in \mathbb{Z}\right\} .
$$

The triple $(V, \tau, \widetilde{V} / \Gamma)$ defines a complex chart for $X_{\Delta}$. The others can be described similarly.

\section{Nonsimple and Nonrational:}

\section{The Regular Icosahedron}

Let $\Delta$ be the regular icosahedron that is dual to the dodecahedron in Section 7 (see Figure 7). Its vertices are given by

$$
\begin{aligned}
& v_{1}=\left(\frac{1}{\phi^{2}}, \frac{1}{\phi}, 0\right), \\
& v_{2}=\left(0, \frac{1}{\phi^{2}}, \frac{1}{\phi}\right), \\
& v_{3}=\left(\frac{1}{\phi}, 0, \frac{1}{\phi^{2}}\right), \\
& v_{4}=\left(-\frac{1}{\phi^{2}}, \frac{1}{\phi}, 0\right), \\
& v_{5}=\left(0,-\frac{1}{\phi^{2}}, \frac{1}{\phi}\right), \\
& v_{6}=\left(\frac{1}{\phi}, 0,-\frac{1}{\phi^{2}}\right), \\
& v_{7}=-v_{1}, \\
& v_{8}=-v_{2}, \\
& v_{9}=-v_{3}, \\
& v_{10}=-v_{4}, \\
& v_{11}=-v_{5}, \\
& v_{12}=-v_{6} .
\end{aligned}
$$

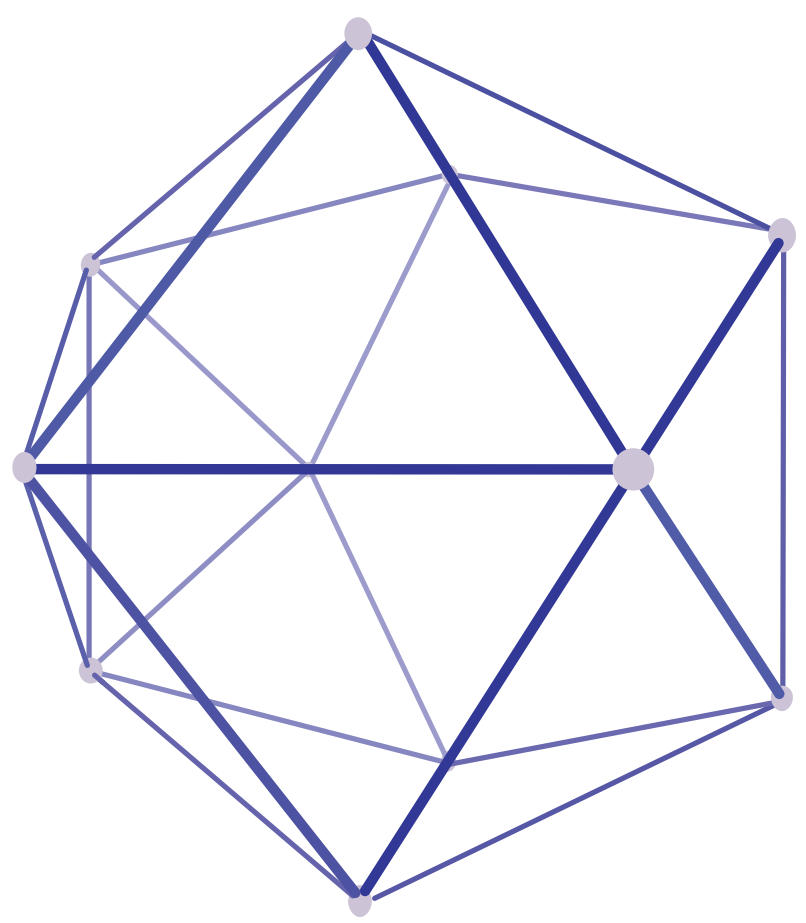

Figure 7: The regular icosahedron.

It is not a rational polytope. However it is quasirational with respect to the quasilattice $B$, known in physics as the bodycentered lattice [18], that is generated by the six vectors

$$
\begin{aligned}
& V_{1}=\left(\phi, \frac{1}{\phi}, 0\right), \\
& V_{2}=\left(0, \phi, \frac{1}{\phi}\right), \\
& V_{3}=\left(\frac{1}{\phi}, 0, \phi\right), \\
& V_{4}=\left(-\phi, \frac{1}{\phi}, 0\right), \\
& V_{5}=\left(0,-\phi, \frac{1}{\phi}\right), \\
& V_{6}=\left(\frac{1}{\phi}, 0,-\phi\right) .
\end{aligned}
$$

Consider in fact the four additional vectors in $B$ given by

$$
\begin{gathered}
V_{7}=-V_{4}-V_{5}-V_{6}=(1,1,1), \\
V_{8}=-V_{1}+V_{3}-V_{5}=(-1,1,1), \\
V_{9}=+V_{1}-V_{2}-V_{6}=(1,-1,1), \\
V_{10}=+V_{2}-V_{3}-V_{4}=(1,1,-1) .
\end{gathered}
$$




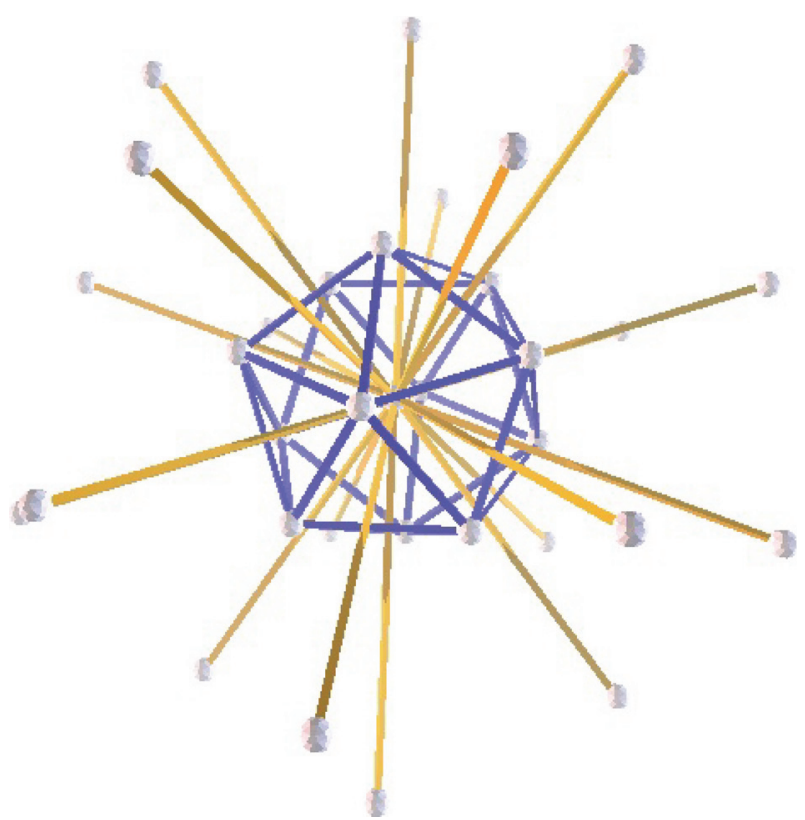

FIGURE 8: The normal vectors $X_{1}, \ldots, X_{20}$.

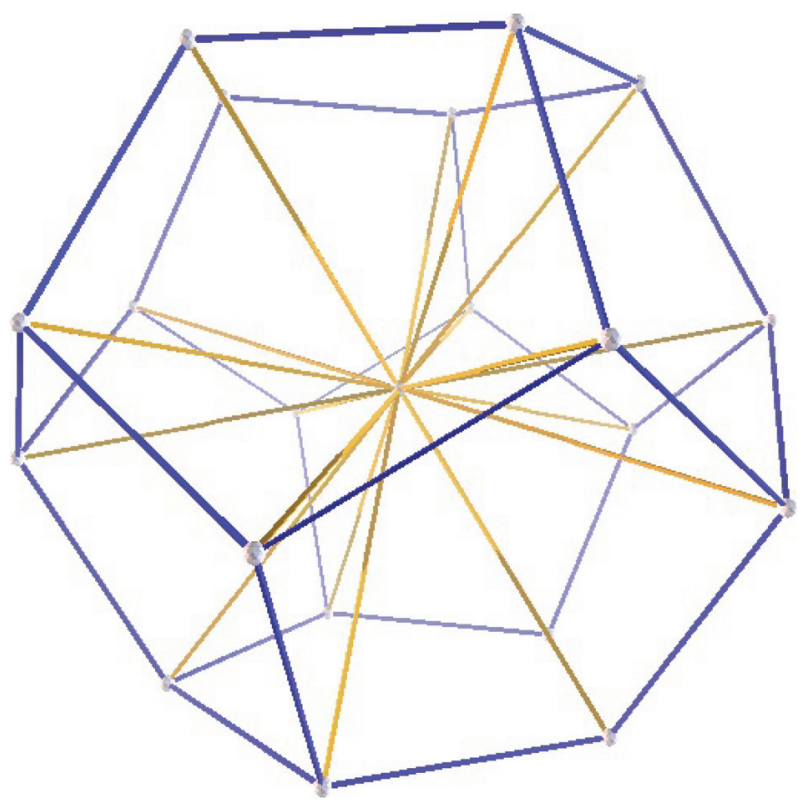

FIgURE 9: The dual dodecahedron.

Then

$$
\Delta=\bigcap_{j=1}^{20}\left\{\mu \in\left(\mathbb{R}^{3}\right)^{*} \mid\left\langle\mu, X_{j}\right\rangle \geq-1\right\},
$$

where $X_{i}=V_{i}, X_{10+i}=-V_{i}, i=1, \ldots, 10$. Notice that the vectors $X_{1}, \ldots, X_{20}$ point to the vertices of the dual regular dodecahedron of Section 7 (see Figures 8 and 9).

The regular icosahedron is not a simple polytope. More precisely, consider the planes

$$
H_{i}=\left\{\mu \in\left(\mathbb{R}^{3}\right)^{*} \mid\left\langle\mu, X_{j}\right\rangle=-1\right\}, \quad i=1, \ldots, 20,
$$

TABLE 2

\begin{tabular}{lc}
\hline Plane & Vertices \\
\hline$H_{1}$ & $\left(v_{7}, v_{9}, v_{12}\right)$ \\
$H_{2}$ & $\left(v_{7}, v_{8}, v_{10}\right)$ \\
$H_{3}$ & $\left(v_{8}, v_{9}, v_{11}\right)$ \\
$H_{4}$ & $\left(v_{3}, v_{6}, v_{10}\right)$ \\
$H_{5}$ & $\left(v_{1}, v_{4}, v_{11}\right)$ \\
$H_{6}$ & $\left(v_{2}, v_{5}, v_{12}\right)$ \\
$H_{7}$ & $\left(v_{7}, v_{8}, v_{9}\right)$ \\
$H_{8}$ & $\left(v_{6}, v_{8}, v_{10}\right)$ \\
$H_{9}$ & $\left(v_{4}, v_{9}, v_{11}\right)$ \\
$H_{10}$ & $\left(v_{5}, v_{7}, v_{12}\right)$ \\
$H_{11}$ & $\left(v_{1}, v_{3}, v_{6}\right)$ \\
$H_{12}$ & $\left(v_{1}, v_{2}, v_{4}\right)$ \\
$H_{13}$ & $\left(v_{2}, v_{3}, v_{5}\right)$ \\
$H_{14}$ & $\left(v_{4}, v_{9}, v_{12}\right)$ \\
$H_{15}$ & $\left(v_{5}, v_{7}, v_{10}\right)$ \\
$H_{16}$ & $\left(v_{6}, v_{8}, v_{11}\right)$ \\
$H_{17}$ & $\left(v_{1}, v_{2}, v_{3}\right)$ \\
$H_{18}$ & $\left(v_{2}, v_{4}, v_{12}\right)$ \\
$H_{19}$ & $\left(v_{3}, v_{5}, v_{10}\right)$ \\
$H_{20}$ & $\left(v_{1}, v_{6}, v_{11}\right)$ \\
\hline &
\end{tabular}

supporting the facets of $\Delta$. Notice that each such plane contains exactly three vertices as shown in Table 2.

Furthermore, each vertex is given by the intersection of five planes:

$$
\begin{aligned}
& v_{1}=H_{5} \cap H_{11} \cap H_{12} \cap H_{17} \cap H_{20}, \\
& v_{2}=H_{6} \cap H_{12} \cap H_{13} \cap H_{17} \cap H_{18}, \\
& v_{3}=H_{4} \cap H_{11} \cap H_{13} \cap H_{17} \cap H_{19}, \\
& v_{4}=H_{5} \cap H_{9} \cap H_{12} \cap H_{14} \cap H_{18}, \\
& v_{5}=H_{6} \cap H_{10} \cap H_{13} \cap H_{15} \cap H_{19}, \\
& v_{6}=H_{4} \cap H_{8} \cap H_{11} \cap H_{16} \cap H_{20}, \\
& v_{7}=H_{1} \cap H_{2} \cap H_{7} \cap H_{10} \cap H_{15}, \\
& v_{8}=H_{2} \cap H_{3} \cap H_{7} \cap H_{8} \cap H_{16}, \\
& v_{9}=H_{1} \cap H_{3} \cap H_{7} \cap H_{9} \cap H_{14}, \\
& v_{10}=H_{2} \cap H_{4} \cap H_{8} \cap H_{15} \cap H_{19}, \\
& v_{11}=H_{3} \cap H_{5} \cap H_{9} \cap H_{16} \cap H_{20}, \\
& v_{12}=H_{1} \cap H_{6} \cap H_{10} \cap H_{14} \cap H_{18} .
\end{aligned}
$$

Let us now apply the generalized Delzant construction. It is easy to see that the relations

$$
\begin{aligned}
& V_{1}=-\frac{1}{2}\left[\left(\phi+\frac{1}{\phi}\right) V_{4}+\phi V_{5}+\frac{1}{\phi} V_{6}\right], \\
& V_{2}=-\frac{1}{2}\left[\frac{1}{\phi} V_{4}+\left(\phi+\frac{1}{\phi}\right) V_{5}+\phi V_{6}\right],
\end{aligned}
$$




$$
\begin{aligned}
V_{3} & =-\frac{1}{2}\left[\phi V_{4}+\frac{1}{\phi} V_{5}+\left(\phi+\frac{1}{\phi}\right) V_{6}\right], \\
V_{8} & =\frac{1}{2}\left[\frac{1}{\phi} V_{4}-V_{5}-\phi V_{6}\right], \\
V_{9} & =\frac{1}{2}\left[-\phi V_{4}+\frac{1}{\phi} V_{5}-V_{6}\right], \\
V_{10} & =\frac{1}{2}\left[-V_{4}-\phi V_{5}+\frac{1}{\phi} V_{6}\right]
\end{aligned}
$$

imply that the kernel of $\pi, \mathfrak{n}$, is the 17 -dimensional subspace of $\mathbb{R}^{20}$ that is spanned by the vectors

$$
\begin{gathered}
e_{i}+e_{10+i}, \quad i=1, \ldots, 10, \\
e_{1}+\frac{1}{2}\left[\left(\phi+\frac{1}{\phi}\right) e_{4}+\phi e_{5}+\frac{1}{\phi} e_{6}\right], \\
e_{2}+\frac{1}{2}\left[\frac{1}{\phi} e_{4}+\left(\phi+\frac{1}{\phi}\right) e_{5}+\phi e_{6}\right], \\
e_{3}+\frac{1}{2}\left[\phi e_{4}+\frac{1}{\phi} e_{5}+\left(\phi+\frac{1}{\phi}\right) e_{6}\right], \\
e_{8}+\frac{1}{2}\left[-\frac{1}{\phi} e_{4}+e_{5}+\phi e_{6}\right] \\
e_{9}+\frac{1}{2}\left[\phi e_{4}-\frac{1}{\phi} e_{5}+e_{6}\right], \\
e_{10}+\frac{1}{2}\left[e_{4}+\phi e_{5}-\frac{1}{\phi} e_{6}\right] .
\end{gathered}
$$

Since the vectors $X_{i}, i=1, \ldots, 20$, generate the quasilattice $B$, the group $N$ is connected and given by the group $\exp (\mathfrak{n})$. The level set $\Psi^{-1}(0)$ for the moment mapping $\Psi$ with respect to the induced $N$-action on $\mathbb{C}^{20}$ is given by the compact subset of points $\underline{z}$ in $\mathbb{C}^{20}$ such that

$$
\begin{aligned}
\left|z_{i}\right|^{2}+\left|z_{10+i}\right|^{2} & =2 \\
i=1, \ldots, 10, & =2 \phi, \\
\left|z_{1}\right|^{2}+\frac{1}{2}\left[\left(\phi+\frac{1}{\phi}\right)\left|z_{4}\right|^{2}+\phi\left|z_{5}\right|^{2}+\frac{1}{\phi}\left|z_{6}\right|^{2}\right] & =\left.\left.2 \phi\right|^{2}\right|^{2}+\frac{1}{2}\left[\frac{1}{\phi}\left|z_{4}\right|^{2}+\left(\phi+\frac{1}{\phi}\right)\left|z_{5}\right|^{2}+\phi\left|z_{6}\right|^{2}\right]=2 \phi, \\
\left|z_{3}\right|^{2}+\frac{1}{2}\left[\phi\left|z_{4}\right|^{2}+\frac{1}{\phi}\left|z_{5}\right|^{2}+\left(\phi+\frac{1}{\phi}\right)\left|z_{6}\right|^{2}\right] & =2 \phi, \\
\left|z_{7}\right|^{2}+\left|z_{4}\right|^{2}+\left|z_{5}\right|^{2}+\left|z_{6}\right|^{2} & =4, \\
\left|z_{8}\right|^{2}+\frac{1}{2}\left[-\frac{1}{\phi}\left|z_{4}\right|^{2}+\left|z_{5}\right|^{2}+\phi\left|z_{6}\right|^{2}\right] & =2,
\end{aligned}
$$

$$
\begin{aligned}
& \left|z_{9}\right|^{2}+\frac{1}{2}\left[\phi\left|z_{4}\right|^{2}-\frac{1}{\phi}\left|z_{5}\right|^{2}+\left|z_{6}\right|^{2}\right]=2, \\
& \left|z_{10}\right|^{2}+\frac{1}{2}\left[\left|z_{4}\right|^{2}+\phi\left|z_{5}\right|^{2}-\frac{1}{\phi}\left|z_{6}\right|^{2}\right]=2 .
\end{aligned}
$$

By Theorem 12, the quotient $M_{\Delta}=\Psi^{-1}(0) / N$ is stratified by symplectic quasifolds.

From the complex viewpoint, consider the open subset $\mathbb{C}_{\Delta}^{20}$ of $\mathbb{C}^{20}$ obtained by (14) using (73). By Theorem 14 , the quotient $X_{\Delta}=\mathbb{C}_{\Delta}^{20} / / N_{\mathbb{C}}$ is stratified by complex quasifolds.

By Theorem 15, $M_{\Delta}$ and $X_{\Delta}$ can be identified. Their global description is similar to the one given for the symplectic and complex toric spaces corresponding to the octahedron, except that here $D^{3}=\mathbb{R}^{3} / B$ is a quasitorus and the regular stratum is a quasifold.

We describe a chart for the regular part of the complex quotient. We choose the vertex $v_{4}$ and the triple $\{5,9,14\}$. A local model for the following open subset of the regular part

$$
\frac{\left\{\underline{z} \in \mathbb{C}_{\Delta}^{20} \mid z_{i} \neq 0, i \neq 5,9,14,\left(z_{5}, z_{9}, z_{14}\right) \neq(0,0,0)\right\}}{N_{\mathbb{C}}}
$$

is given by $\left(\mathbb{C}^{3} \backslash\{0\}\right) / \Gamma$, where

$$
\Gamma=\exp \{(-\phi h, \phi(h+k+2 l),-\phi k) \mid h, k, l \in \mathbb{Z}\} .
$$

Similarly to what happens for the octahedron, the quotient $X_{\Delta}$ around the singular point corresponding to $v_{4}$ is a cone. We will also have a diagram similar to (52). In this case

$$
\mathfrak{n}(\mathscr{C})=\{(-\phi s-t, \phi(s+t), s,-s-\phi t, t) \mid s, t \in \mathbb{R}\},
$$

while

$N(\mathscr{C})$

$$
\begin{aligned}
& =\exp \{(-\phi s-t, \phi(s+t+2 l), s,-s-\phi t, t) \mid s, t \\
& \in \mathbb{R}, l \in \mathbb{Z}\} .
\end{aligned}
$$

The components of the moment mapping $\Psi$ : $\mathbb{C}^{5} \rightarrow(\mathfrak{n}(\mathscr{C}))^{*}$ with respect to the basis of $(\mathfrak{n}(\mathscr{C}))^{*}$ dual to $(-\phi, \phi, 1,-1,0),(-1, \phi, 0,-\phi, 1)$ are

$$
\begin{aligned}
& -\phi\left|z_{1}\right|^{2}+\phi\left|z_{2}\right|^{2}+\left|z_{3}\right|^{2}-\left|z_{4}\right|^{2}, \\
& -\left|z_{1}\right|^{2}+\phi\left|z_{2}\right|^{2}-\left|z_{4}\right|^{2}+\left|z_{5}\right|^{2} .
\end{aligned}
$$

As in the case of the octahedron, we can find the symplectic and complex toric spaces corresponding to the cone $\mathscr{C}$; these are regular symplectic and complex quasifolds away from the point corresponding to the cone apex. We now describe the local structure of the stratification. Consider the vector

$$
\begin{aligned}
X_{v_{4}} & =2(-1, \phi, 0) \\
& =-\frac{2}{2+\phi}\left(X_{5}+X_{9}+X_{12}+X_{14}+X_{18}\right) \\
& =-V_{1}+V_{2}+V_{3}+V_{4}-V_{5}+V_{6} \in B .
\end{aligned}
$$




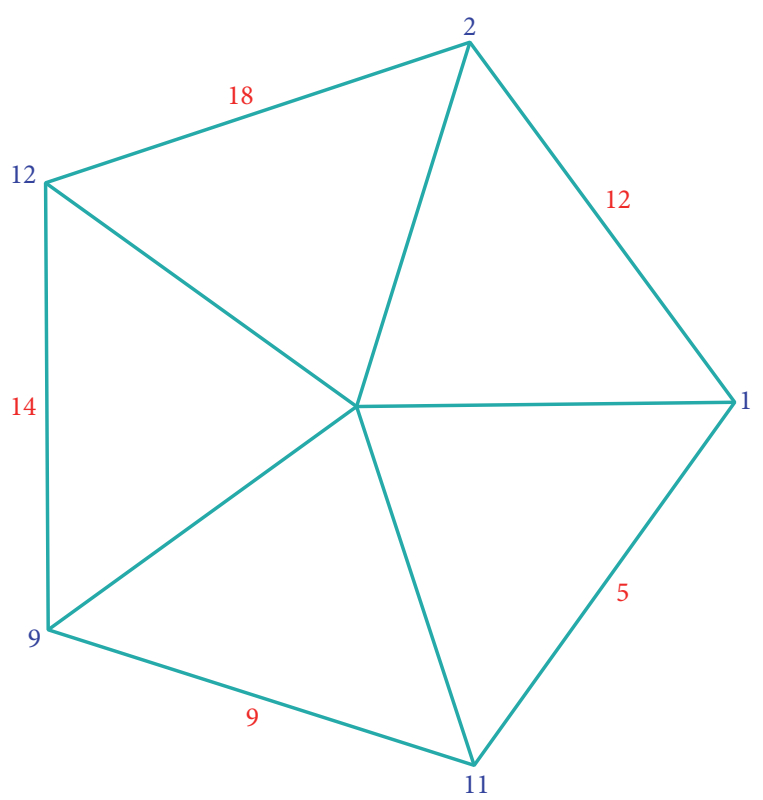

Figure 10: The pentagon $\Delta_{L}$; its vertices and facets are suitably labeled.

The plane $H_{\nu_{4}}=\left\{\mu \in\left(\mathbb{R}^{3}\right)^{*} \mid\left\langle\mu, X_{\nu_{4}}\right\rangle=2 / \phi\right\}$ cuts the icosahedron in the regular pentagon $\Delta_{L}$ whose vertices are given by $\left\{v_{1}, v_{2}, v_{9}, v_{11}, v_{12}\right\}$ (see Figure 10). Let $\xi_{v_{4}}=(-1 / \phi(2+\phi), 1 /(2+\phi), 0)$ be a point in the cutting plane $H_{v_{4}}$. The rigid motion $R+\xi_{v_{4}}:\left(\mathbb{R}^{3}\right)^{*} \rightarrow\left(\mathbb{R}^{3}\right)^{*}$, where $R$ is the rotation

$$
\left(\begin{array}{ccc}
\frac{\phi}{\sqrt{2+\phi}} & -\frac{1}{\sqrt{2+\phi}} & 0 \\
\frac{1}{\sqrt{2+\phi}} & \frac{\phi}{\sqrt{2+\phi}} & 0 \\
0 & 0 & 1
\end{array}\right)
$$

maps the $x z$-plane into $H_{v_{4}}$. We obtain that $\Delta_{L}$ equals

$$
\begin{aligned}
& \bigcap_{i=5,9,12,14,18}\left\{\mu=\left(\mu_{1}, 0, \mu_{3}\right)\right. \\
& \left.\quad \in\left(\mathbb{R}^{3}\right)^{*} \mid\left\langle R(\mu)+\xi_{v_{4}}, X_{i}\right\rangle \geq-1\right\} .
\end{aligned}
$$

This gives

$$
\begin{aligned}
\Delta_{L} & =\bigcap_{i=5,9,12,14,18}\left\{\left(\mu_{1}, \mu_{3}\right) \in\left(\mathbb{R}^{2}\right)^{*} \mid\left\langle\left(\mu_{1}, \mu_{3}\right), Y_{i}\right\rangle\right. \\
& \left.\geq-\frac{2}{2+\phi}\right\},
\end{aligned}
$$

with

$$
\begin{aligned}
& Y_{5}=\left(-\frac{\phi}{\sqrt{2+\phi}}, \frac{1}{\phi}\right), \\
& Y_{9}=\left(\frac{1}{\phi \sqrt{2+\phi}}, 1\right),
\end{aligned}
$$

$$
\begin{aligned}
& Y_{12}=\left(-\frac{\phi}{\sqrt{2+\phi}},-\frac{1}{\phi}\right), \\
& Y_{14}=\left(\frac{2}{\sqrt{2+\phi}}, 0\right), \\
& Y_{18}=\left(\frac{1}{\phi \sqrt{2+\phi}},-1\right) .
\end{aligned}
$$

The above vectors are obtained from $X_{5}, X_{9}, X_{12}, X_{14}, X_{18}$ by rotating with $R^{t}$, the transpose of $R$, and then projecting onto the $x z$-plane. They generate the pentagonal quasilattice $B_{L}$ given by rotating and projecting the icosahedral quasilattice $B$ onto the $x z$-plane. In fact, it is easy to check that $X_{5}, X_{9}, X_{12}, X_{14}, X_{18}, X_{v_{4}}$ generate $B$ and, moreover, that $R^{t}\left(X_{v_{4}}\right)$ is parallel to $(0,1,0)$. Now we apply the generalized Delzant procedure to $\Delta_{L}$ with respect to the quasilattice $B_{L}$ and the vectors $Y_{i}$ above. Again here $N\left(\Delta_{L}\right)$ is connected and is given by

$$
\begin{aligned}
& \exp (\{(-\phi r-\phi s-t, r+\phi s+\phi t, s,-\phi r-s-\phi t, t) \mid r, s, t \\
& \quad \in \mathbb{R}\}) .
\end{aligned}
$$

The level set $\Psi^{-1}(0)$ for the moment mapping $\Psi$ with respect to the induced $N\left(\Delta_{L}\right)$-action on $\mathbb{C}^{5}$ is therefore the compact submanifold of $\mathbb{C}^{5}$ given by the points $\underline{z}$ in $\mathbb{C}^{5}$ such that

$$
\begin{array}{r}
\phi\left|z_{1}\right|^{2}-\left|z_{2}\right|^{2}+\phi\left|z_{4}\right|^{2}=\frac{2}{\phi}, \\
-\phi\left|z_{1}\right|^{2}+\phi\left|z_{2}\right|^{2}+\left|z_{3}\right|^{2}-\left|z_{4}\right|^{2}=0, \\
-\left|z_{1}\right|^{2}+\phi\left|z_{2}\right|^{2}-\phi\left|z_{4}\right|^{2}+\left|z_{5}\right|^{2}=0 .
\end{array}
$$

We thus obtain the 6-dimensional symplectic quasifold $M_{\Delta_{L}}=\Psi^{-1}(0) / N\left(\Delta_{L}\right)$. From the complex viewpoint, the toric quasifold $X_{\Delta_{L}}$ is given by the quotient $\mathbb{C}_{\Delta_{L}}^{5} / N_{\mathbb{C}}\left(\Delta_{L}\right)$, where the open subset $\mathbb{C}_{\Delta_{L}}^{5}$ can be easily determined from Figure 10. We know that $N(\mathscr{C})$ is a subgroup of $N\left(\Delta_{L}\right)$; in fact it is given by

$$
\begin{aligned}
& \{\exp (-\phi r-\phi s-t, r+\phi s+\phi t, s,-\phi r-s-\phi t, t) \\
& \left.\quad \in N\left(\Delta_{L}\right) \mid r=-\frac{2}{\phi} l, \text { with } l \in \mathbb{Z}\right\} .
\end{aligned}
$$

Interestingly, the quotient $N\left(\Delta_{L}\right) / N(\mathscr{C})$ is the quasitorus $\mathbb{R} / 2 \phi \mathbb{Z}$. Thus, in this case, the fibers of the projections $p$ and $p_{\mathbb{C}}$ of diagram (52) are given by

$$
\frac{N_{\mathbb{C}}\left(\Delta_{L}\right)}{N_{\mathbb{C}}(\mathscr{C})} \simeq \mathbb{R}_{>0} \times \frac{\mathbb{R}}{2 \phi \mathbb{Z}} .
$$

\section{Conflicts of Interest}

The authors declare that there are no conflicts of interest regarding the publication of this paper. 


\section{Acknowledgments}

This research was partially supported by Grant PRIN 2010NNBZ78_012 (MIUR, Italy). All 3D pictures were drawn using ZomeCAD.

\section{References}

[1] T. Delzant, "Hamiltoniens périodiques et images convexes de l'application moment," Bulletin de la Société Mathématique de France, vol. 116, no. 3, pp. 315-339, 1988.

[2] W. Fulton, Introduction to Toric Varieties, vol. 131 of Annals of Mathematics Studies, Princeton University Press, Princeton, NJ, USA, 1993.

[3] M. Audin, The Topology of Torus Actions on Symplectic Manifolds, vol. 93 of Progress in Mathematics, Birkhäuser, Basel, Switzerland, 1991.

[4] D. A. Cox, "The homogeneous coordinate ring of a toric variety," Journal of Algebraic Geometry, vol. 4, pp. 17-50, 1995.

[5] E. Prato, "Symplectic toric geometry and the regular dodecahedron," Journal of Mathematics, Art. ID 967417, 5 pages, 2015.

[6] E. Prato, "Simple non-rational convex polytopes via symplectic geometry," Topology. An International Journal of Mathematics, vol. 40, no. 5, pp. 961-975, 2001.

[7] F. Battaglia and E. Prato, "Generalized toric varieties for simple nonrational convex polytopes," International Mathematics Research Notices, no. 24, pp. 1315-1337, 2001.

[8] F. Battaglia and E. Prato, "Nonrational, nonsimple convex polytopes in symplectic geometry," Electronic Research Announcements of the American Mathematical Society, vol. 8, pp. 29-34, 2002.

[9] F. Battaglia, "Convex polytopes and quasilattices from the symplectic viewpoint," Communications in Mathematical Physics, vol. 269, no. 2, pp. 283-310, 2007.

[10] F. Battaglia, "Geometric spaces from arbitrary convex polytopes," International Journal of Mathematics, vol. 23, no. 1, Article ID 1250013, 39 pages, 2012.

[11] G. M. Ziegler, Lectures on Polytopes, vol. 152 of Graduate Texts in Mathematics, Springer, New York, NY, USA, 1995.

[12] F. Battaglia and E. Prato, "The symplectic Penrose kite," Communications in Mathematical Physics, vol. 299, no. 3, pp. 577-601, 2010.

[13] V. Guillemin, Moment Maps and Combinatorial Invariants of Hamiltonian $\mathrm{T}^{n}$-Spaces, vol. 122 of Progress in Mathematics, Birkhäuser Boston, Boston, Mass, USA, 1994.

[14] M. Goresky and R. MacPherson, Stratified Morse Theory, Springer, Berlin, Germany, 1988.

[15] R. Sjamaar and E. Lerman, "Stratified symplectic spaces and reduction," Annals of Mathematics. Second Series, vol. 134, no. 2, pp. 375-422, 1991.

[16] D. M. Burns, V. W. Guillemin, and E. M. Lerman, "Toric symplectic singular spaces I: isolated singularities," The Journal of Symplectic Geometry, vol. 3, no. 4, pp. 531-543, 2005.

[17] D. Burns, V. Guillemin, and E. Lerman, "Kähler metrics on singular toric varieties," Pacific Journal of Mathematics, vol. 238, no. 1, pp. 27-40, 2008.

[18] D. S. Rokhsar, N. D. Mermin, and D. C. Wright, "Rudimentary quasicrystallography: the icosahedral and decagonal reciprocal lattices," Physical Review. B, vol. 35, pp. 5487-5495, 1987. 


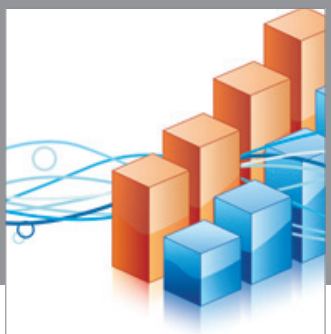

Advances in

Operations Research

vatem alat4

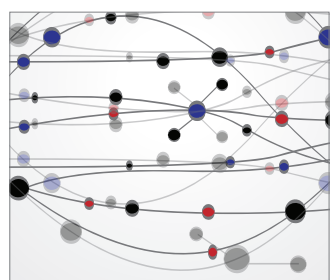

\section{The Scientific} World Journal
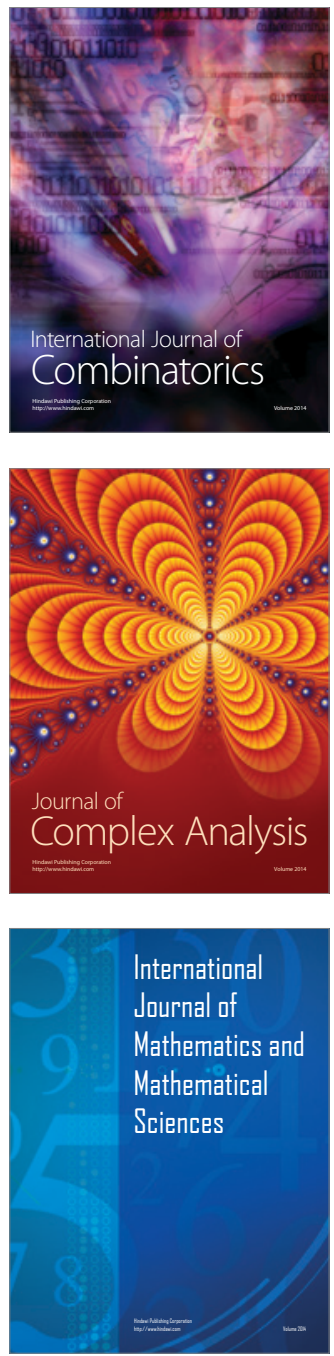
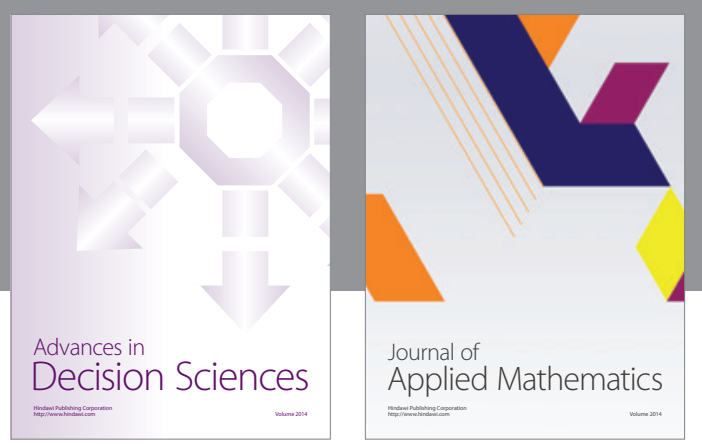

Algebra

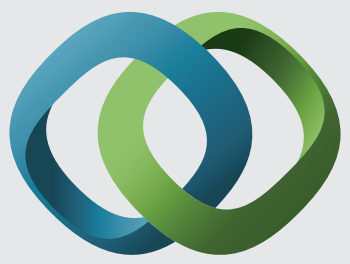

\section{Hindawi}

Submit your manuscripts at

https://www.hindawi.com
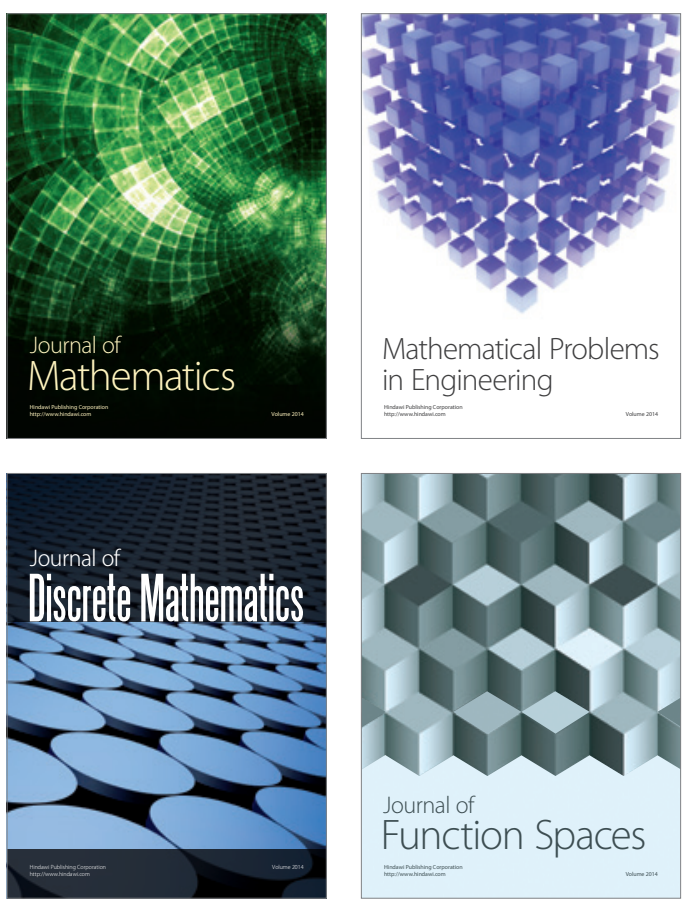

Mathematical Problems in Engineering
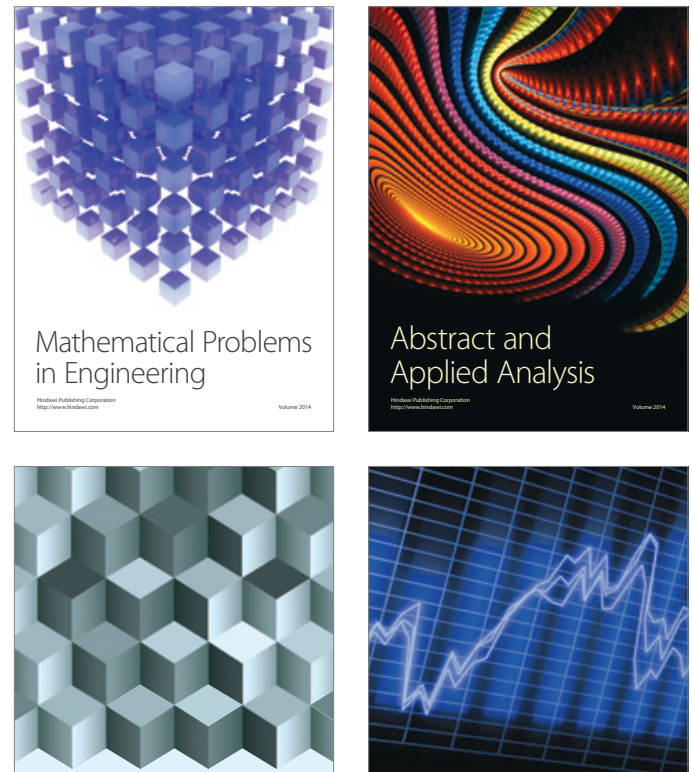

Journal of

Function Spaces

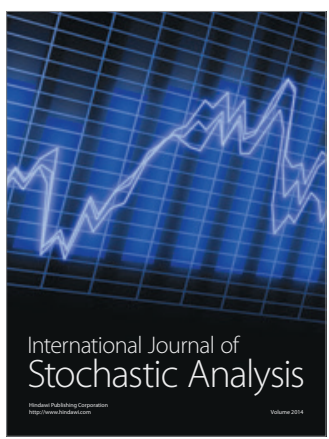

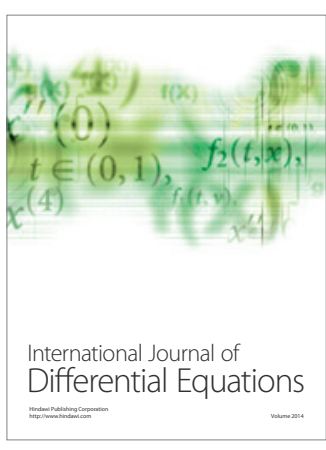
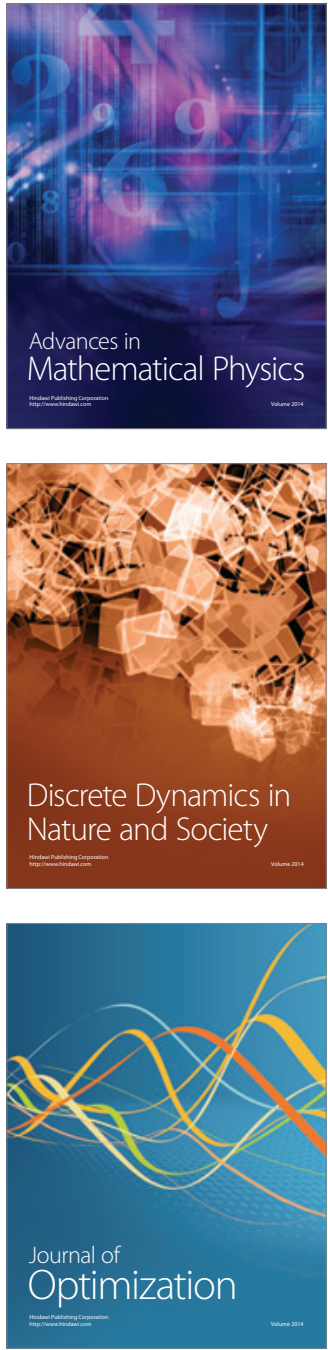